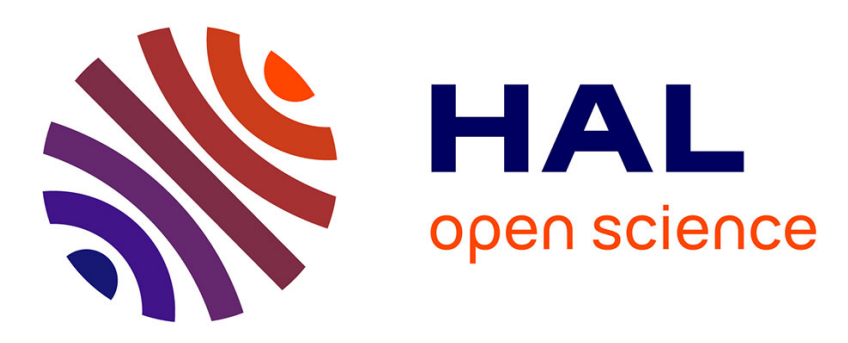

\title{
ALOHA: an Advanced LOwer Hybrid Antenna coupling code
}

Julien Hillairet, Damien Voyer, Annika Ekedahl, Marc Goniche, Michal Kazda, Orso Meneghini, Daniele Milanesio, Mélanie Preynas

\section{> To cite this version:}

Julien Hillairet, Damien Voyer, Annika Ekedahl, Marc Goniche, Michal Kazda, et al.. ALOHA: an Advanced LOwer Hybrid Antenna coupling code. Nuclear Fusion, 2010, 50 (12), pp.125010. 10.1088/0029-5515/50/12/125010 . hal-01005510

\section{HAL Id: hal-01005510 https://hal.science/hal-01005510}

Submitted on 13 Jun 2014

HAL is a multi-disciplinary open access archive for the deposit and dissemination of scientific research documents, whether they are published or not. The documents may come from teaching and research institutions in France or abroad, or from public or private research centers.
L'archive ouverte pluridisciplinaire HAL, est destinée au dépôt et à la diffusion de documents scientifiques de niveau recherche, publiés ou non, émanant des établissements d'enseignement et de recherche français ou étrangers, des laboratoires publics ou privés. 


\title{
ALOHA: an Advanced LOwer Hybrid Antenna coupling code
}

\author{
J. Hillairet ${ }^{1}$, D. Voyer ${ }^{2}$, A. Ekedahl ${ }^{1}$, M. Goniche ${ }^{1}$, M. Kazda ${ }^{1}$, \\ O. Meneghini ${ }^{3}$, D. Milanesio ${ }^{4}$, M. Preynas ${ }^{1}$ \\ 1 CEA, IRFM, F-13108 Saint Paul-lez-Durance, France. \\ 2 Centrale Lyon, 69134 Ecully, France. \\ 3 MIT Plasma Science and Fusion Center, Cambdrige, USA \\ ${ }^{4}$ Politecnico Di Torino, Dipartimento di Elettronica, Torino, Italy. \\ E-mail: julien.hillairet@cea.fr
}

\begin{abstract}
The Advanced LOwer Hybrid Antenna Code (ALOHA) code, has been developed to improve the modeling of the coupling of lower hybrid $(\mathrm{LH})$ waves from the antenna to a cold inhomogeneous plasma while keeping a fast tool. In contrast to the previous code SWAN (that only described the interaction of the slow wave between the waveguides and the plasma in a 1D model), the equations are now solved in 2D including the contribution of both the slow and fast waves, with a low computational cost. This approach is completed either by a full-wave computation of the antenna that takes into account its detailed geometry or by a mode-matching code dedicated to multijunctions modeling, which is convenient in preliminary design phases. Moreover, ALOHA can treat more realistic scrape-off layers in front of the antenna, by using a two-layer electron density profile. The ALOHA code has been compared with experimental results from Tore Supra LH antennas of different geometries, as well as benchmarked against other LH coupling codes, with very good results. Once validated, ALOHA has been used as a support for the design of COMPASS and ITER LH antennas and has shown to be a fast and reliable tool for LH antenna design.
\end{abstract}

Keywords: ALOHA, Lower Hybrid, Coupling code, Current Drive, Grill, Multijunction, PAM, Linear LH coupling theory 


\section{Introduction}

Lower Hybrid (LH) waves have been known for years to be an efficient way to drive current in Tokamaks since the LH waves interact strongly with electrons via Landau damping. This feature is well-suited to address long pulses issues, to save magnetic flux during the current ramp-up phase or for steady-state and hybrid scenarios [1]. Thus, LH Current Drive (LH-CD) is a key element of a large number of experiments (JET, Alcator C-Mod, FTU, HT-7, Tore Supra) and is planned to be integrated on recent devices (COMPASS, EAST, KSTAR, SST1). In ITER, LH-CD is due to be commissioned and used for the $\mathrm{Q}=5$ steady-state and efficient off-axis CD [2]. However, the LH coupling depends on the edge plasma conditions and a weaker coupling may modify the electric field pattern of the launched wave and therefore affect the CD efficiency.

Up to now, most of the theoretical work has been focused on plasma coupling properties of the antenna based on a description complying with the linear theory [3, 4]. Best known linear coupling codes used to design present operating antennas are Slow Wave ANtenna (SWAN) [5, 6] or GRILL3D [7]. In these codes, the toroidal lines of waveguides are assumed to be infinite in the poloidal direction and are described using a modal expansion. The modal description of the field makes possible to simulate large antennas with a moderate computing cost but the code cannot handle realistic geometry.

More recently, Torino Politecnico Lower Hybrid Antenna (TOPLHA)[8, 9], a boundary element code in which the antenna is discretized using linear vector functions defined over a triangular surface mesh, has been developed to address the problem of LH wave coupling including the toroidal curvature of the plasma and the realistic shape of the antenna. Some finite elements method (FEM) commercial RF software [10] are now able to treat materials with generic temporally-dispersive dielectric tensors. This leaves absolute freedom in the description of the antenna geometry and of the plasma within the collisional cold plasma approximation[11]. In particular, the FEM approach permits spatial variations of any given quantity allowing for example to specify realistic magnetic equilibrium, arbitrary density profiles, poloidal and toroidal asymmetries, density fluctuations, etc. However, taking into account realistic meshes in a large antenna increases drastically the computation time or the required computational power. This latter point is inconsistent with intensive use, for example in daily experiments when one has to evaluate the effect of modules tripping on the radiated spectrum or during antenna design stage, where many calculations may be expected. In order to interpret and improve present day experiments and to design future antennas, advanced but fast and convenient modeling tools are required.

In order to improve the antenna-plasma coupling description of SWAN code while keeping its low computational cost, a code named Advanced LOwer Hybrid Antenna (ALOHA) has been developed. In ALOHA, LH antennas may be modeled by any RF commercial software or homemade code, in order to take into account exact RF properties of the structure. For optimization process, ALOHA also provides a plug-in named HAMAC (Hybrid Antenna Modeling for the ALOHA Code), which purpose is to compute the RF properties of a multijunction antenna using a mode-matching technique. The plasma coupling is calculated from a fast classical 1D modeling that describes the effect of the slow wave only[12] or from a more advanced 2D modeling that implies the contribution of both fast and slow waves[3, 4, 7]. These calculations are based on the linear cold plasma theory and non-linear effects, such as thermal effects ("warm" dielectric tensor) and ponderomotive force have not been taken into account in the present analysis. However, some specific developments have been brought in one hand, to describe a more realistic electron density profiles taking into account the close environment of LH antennas and in the other hand, to avoid computational difficulties 
when the antenna is large. These assumptions let ALOHA to solve a case in a couple of seconds up to 2 hours on a desktop computer, depending on the number of waveguides of the antenna and the plasma model chosen. By comparison, finite element formulations require high-end or super-computers to be solved.

This paper is divided in 4 sections. In Sec.2, the key elements of the linear coupling theory are recalled and some ALOHA specificities are detailed, such as the use of generalized scattering matrix use, the 2D grill modeling and the two electron density gradients profile. In order to show the interest of the ALOHA code, Sec.3 is devoted to experimental comparisons and simulation benchmarks on Tore Supra antennas. Some new LH antenna designs which have been realized using ALOHA for the COMPASS tokamak and for ITER are given in Sec.4. A discussion on the ALOHA integration to the EFDA Integrated Tokamak Modeling Task Force (ITM-TF) is given into the conclusion.

\section{Main assumptions}

\subsection{Network description}

The antenna of present experiments (JET, Alcator C-Mod, FTU, HT-7, Tore Supra, etc.) are designed to launch an asymmetric parallel wavenumber spectrum where most of the power is generated at a low parallel refractive index $\left|n_{\|}\right| \approx 2$. This is usually achieved by phasing the forward waves in waveguides which are stacked along the static magnetic field. This phasing can be obtained externally in the transmission line feeding of the antenna [13,14, 15] or directly inside the antenna when E-plane power dividers - usually called "multijunction" [6] - are associated with built-in phase shifters[16, 17, 18]. In this paper, the term "module" refers to the unit of the antenna composed of one "input" waveguide fed by the power source and one or several "output" waveguides facing the plasma, and the "grill" refers to the plane containing all the open ended waveguides of the antenna. A complete LH antenna can be made of many different modules, stacked in the toroidal or poloidal directions. A simple example is depicted in figure 1.

In the ALOHA code, the plasma coupling of a LH grill antenna is split in two parts: the modeling of the modules and the modeling of the grill in front of the plasma.

Firstly, the electromagnetic characterization of a module is obtained using some RF software or code, which results in the calculation of the scattering matrix of a module $\mathbb{S}_{\text {module }}$ in which only the propagating modes are taken into account. This matrix quantifies the coupling between all inputs and outputs of the RF structure[19].

Secondly, the coupling to the plasma of all the output waveguides that compose the grill is considered. At this stage, evanescent modes excited at the end of the waveguides are also taken in account. Thus, the grill is characterized by a $N$-port network where $N=N_{\text {wg }} \times N_{\text {modes }}$ is the number of ports, $N_{\mathrm{wg}}$ the total number of output waveguides and $N_{\text {modes }}$ the number of electromagnetic modes in each waveguide. A scattering matrix $\mathbb{S}_{\text {grill/plasma }}$ of dimensions $N \times N$ is then derived using the approach detailed in Section 2.3: this matrix quantifies the coupling between the different ports. In such a modeling, each mode is associated to a port [20]; a port can coincide either with the principal mode that propagate inside an output waveguide or with a higher order mode excited at the end of an output waveguide.

Finally, following the well known network approach, both modeling are combined: the ports in the scattering matrices $\mathbb{S}_{\text {module }}$ and $\mathbb{S}_{\text {grill/plasma }}$ that correspond to the same mode in a waveguide are identified and the global response of the antenna can then be extracted and directly 
compared to experimental data.

For a better understanding, let us consider the example of the antenna shown in figure 1 . It is composed of one toroidal line of waveguides shared into two modules. Each module is a multijunction in which a large input feeding waveguide is split into four smaller output waveguides; the output waveguides are said to be active waveguides since they are connected to the input waveguide inside the module. The modules are separated by a short circuited waveguide; this waveguide is isolated and consequently is said to be passive. Moreover, there is also one passive waveguide on each side of the antenna. Each module is characterized by a scattering matrix $\mathbb{S}_{\text {module1 }}$ and $\mathbb{S}_{\text {module2 }}$

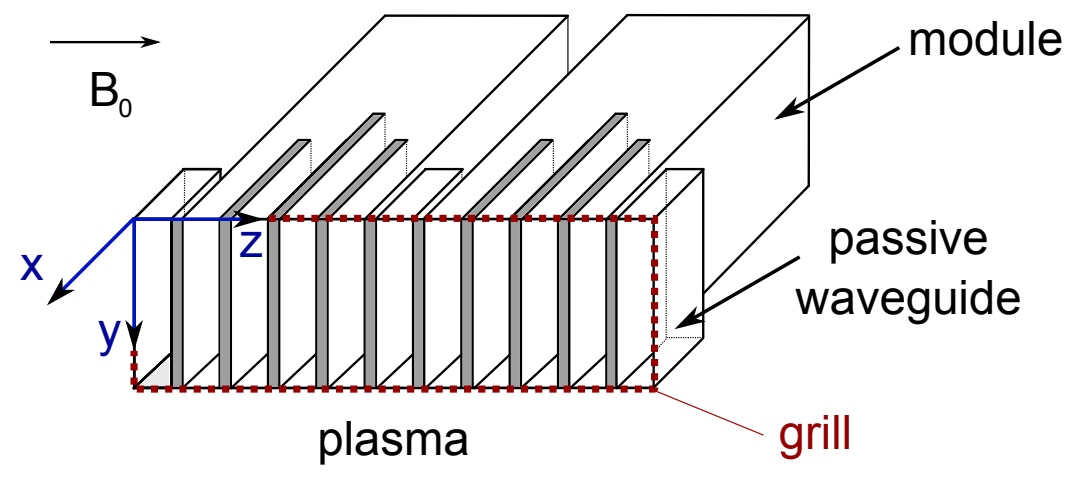

Figure 1. A typical LH (multijunction) antenna structure. In this example, the antenna is made of two multijunction modules stacked in the toroidal $(z)$ direction. One passive waveguide is inserted between both modules and on each side of the antenna.

Suppose that, in front of the plasma, two modes are excited inside the output waveguides: the principal mode $\mathrm{TE}_{10}$ and the evanescent mode $\mathrm{TM}_{11}$. The network modeling of this problem is given in figure 2. In that representation, a port in a N-port network is described by a terminal. Thus, each of both modules is characterized by a 5-port network ( 1 input waveguide $\times 1$ mode + 4 active waveguides $\times 1$ mode) and the coupling of the grill with the plasma is modeled by a 22-port network ( 8 active waveguides $\times 2$ modes +3 passive waveguides $\times 2$ modes). The terminals in the 22 -port network that coincide with the principal mode of the active waveguides in the modules are connected to the corresponding terminals in the 5 -port network. The terminals in the 22-port network that coincide with the principal mode of the passive waveguides are shunt on a piece of transmission line ended by a short-circuit to model the reflection of the wave. The terminals in the 22-port network that coincide with the evanescent modes are shunt on the imaginary mode impedance of the output waveguide, which is equivalent to consider that those modes carry reactive energy in a waveguide of infinite length. Finally, the antenna reduces to a 2-port network in which the ports correspond to the input feeding waveguide of both modules and the global scattering matrix of the antenna is a $2 \times 2$ matrix ( $\mathbb{S}_{\text {access }}$ ). This formalism allows the code to fully take into account the coupling of the modes in the waveguides.

\subsection{Module modeling}

Lower Hybrid experiments show that the coupling efficiency not only depends on the plasma configuration but also on the antenna structure. Antennas are often designed in order to minimize 


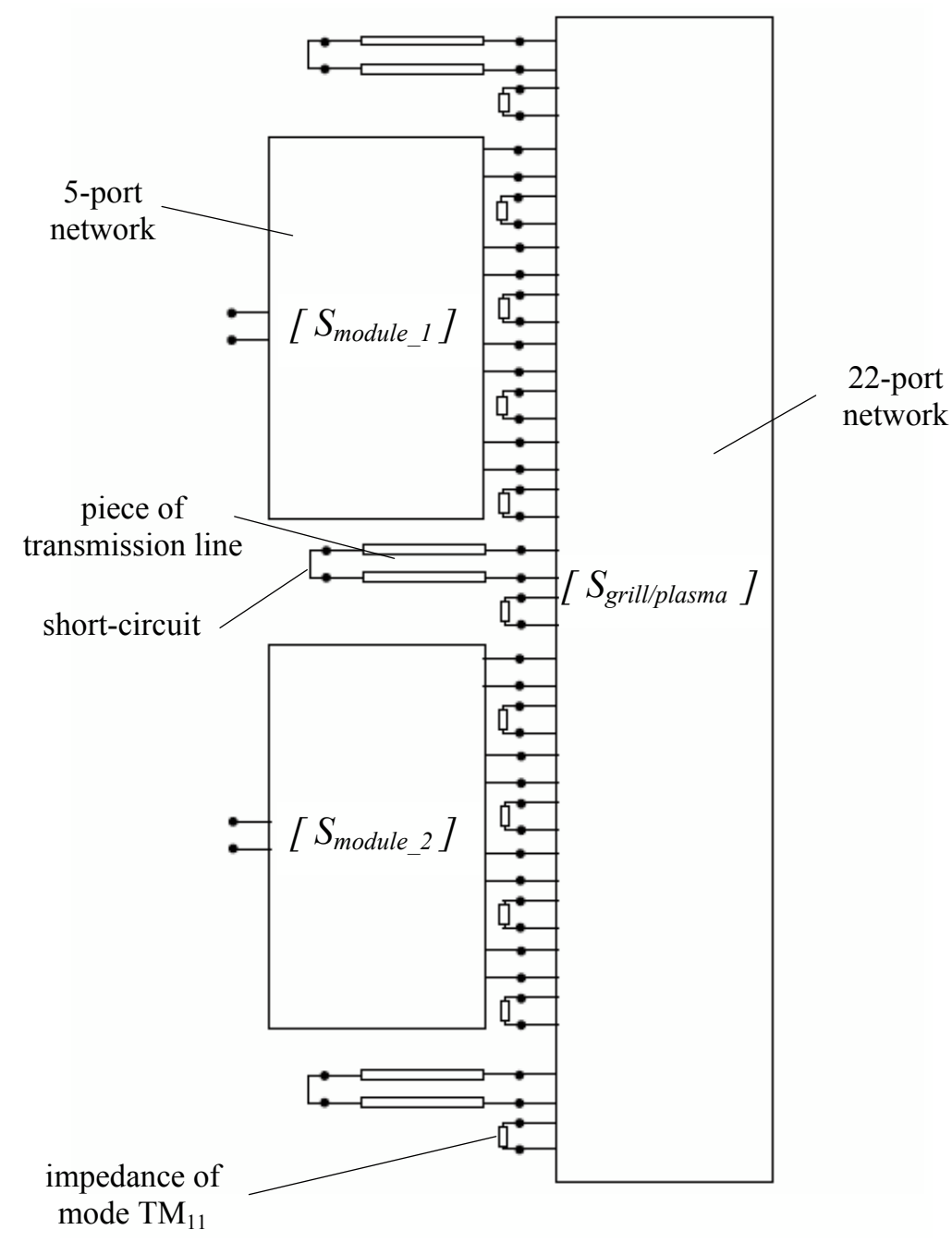

Figure 2. Illustration of the modeling of the antenna described in figure 1 using the N-port concept.

the reflected power from the plasma to the RF sources, thus limiting the need of complex and expensive klystron protection systems. Multijunction concept is frequently used in tokamaks in order to create large waveguide arrays. The necessary phase shifts between adjacent waveguides used to launch non-symmetrical parallel wavenumber spectrum is usually obtained in the design by adjusting the relative guide heights[21]. These built-in phase shifters create multiple passages of the waves through the structure, which lead to a reduction of the reflected power to the RF sources as well as an increase of the electric field strength or an increase of secondary peaks in the radiated power spectrum. Thus, the $\mathrm{RF}$ design of such antennas is less straight-forward than simple open-ended waveguides and an accurate $\mathrm{RF}$ characterization is required to analyze the experimental data. 
The coupling efficiency requires the measurement of the forward and reflected electric fields in all waveguides which in principle can be done but requires the installation under vacuum of a very large number of probes[22, 23]. For an easier maintenance of the RF system, the use of a single bi-directional coupler at the module input in the pressurized transmission line is usually preferred. With this scheme, the electric field map at the plasma-antenna interface, needed to compute the launched parallel index $n_{\|}$, is only accessible by computation. In order to directly compare theoretical coupling predictions to experimental RF measurements, the RF description of each module have to be considered incorporating all the RF components in line from the plasma up to the RF probes location. Thus, the realistic wave propagation inside each module, through multijunctions and power splitters (such as hybrid junctions, magic tees or mode converters) has to be taken into account.

Commercial RF softwares, for example ANSYS-Ansoft HFSS or CST Studio, are convenient tools to design and optimize such components. In ALOHA, the scattering matrix calculated by these codes that describe the modules of the antenna can be directly used as input. Moreover, the HAMAC (Hybrid Antenna Modeling for the ALOHA Code) code has been developed as a plug-in to ALOHA for the design and optimization of simple multijunctions which may not required the use of such complex and advanced software.

In HAMAC, the electromagnetic field is described as a sum of propagative and evanescent modes. Junctions between interior elements such as bi-junction, width and height reductions for phase shifters (E/H-plane discontinuities) or tapers are calculated using a mode-matching technique[20, §8.2]. This technique consists in expressing the continuity of transverse fields at the interface between two waveguides of different cross-section, and uses the orthogonality properties of modes to build and then solve a linear system[24]. The matching between all the elements composing the complete antenna is made using the standard network analysis (see for instance review paper [25]). As a result, the HAMAC plug-in calculates the scattering matrix of a multijunction module. The HAMAC use is illustrated in Sec.4.1 with the example of some designs trials for a new COMPASS LH antenna.

Once the antenna's module scattering matrices calculated $\left(\mathbb{S}_{\text {module }}\right)$, they are used as input for ALOHA to simulate the coupling between the modules and the plasma, as explained in the next sections.

\subsection{Grill-plasma modeling}

2.3.1. Plasma description Lets consider the geometry shown in figure 1. The static magnetic field $\mathbf{B}_{0}$ is assumed to be in the $z$ direction and vary as well as the plasma density in $x$ direction. This hypothesis is specifically valid for large tokamak such as ITER or tokamak with high aspect ratio in which the angle between the magnetic field and the narrow waveguide is small. The interface between waveguides and plasma is set at $x=0$. The plasma is considered homogeneous in $y$ and $z$ directions. The electromagnetic field scattered by the antenna is supposed to be dissipated far away from the coupling region so that the coupling problem is treated as a problem of radiation in a semi-infinite medium independently of the absorption in the core plasma. In such a modeling, the propagation into the plasma core is not calculated, meaning that effects such as mode conversion are not taken into account. From Maxwell's equations, the electromagnetic field in the absence of source satisfies the vector wave equation in the $+j \omega t$ time harmonic convention:

$$
\left(\nabla \times \nabla \times-k_{0}^{2} \mathbb{K} \cdot\right) \mid \begin{aligned}
& \mathbf{E}(\mathbf{r}) \\
& \mathbf{H}(\mathbf{r})
\end{aligned}=0
$$


where $\mathbf{E}$ and $\mathbf{H}$ are the electric and magnetic fields, $\mathbf{r}=x \hat{\mathbf{x}}+y \hat{\mathbf{y}}+z \hat{\mathbf{z}}$ is the space vector coordinates and $k_{0}=\frac{\omega}{c}$ the free-space wavenumber. In the vicinity of the grill, the plasma is described by its (normalized) cold dielectric tensor $\mathbb{K}$ which is given, following Stix notation[26, 27], by:

$$
\mathbb{K}(x, \omega)=\left[\begin{array}{ccc}
S & j D & 0 \\
-j D & S & 0 \\
0 & 0 & P
\end{array}\right]
$$

where parameters $S, D$ and $P$ which depend on radial position $x$ and wave frequency, are defined by:

$$
\begin{aligned}
& S(x, \omega)=1-\sum_{s} \frac{\omega_{p, s}^{2}}{\omega^{2}-\Omega_{c, s}^{2}} \\
& D(x, \omega)=\sum_{s} \frac{\Omega_{c i}}{\omega} \frac{\omega_{p, s}^{2}}{\omega^{2}-\Omega_{c, s}^{2}} \\
& P(x, \omega)=1-\sum_{s} \frac{\omega_{p, s}^{2}}{\omega^{2}}
\end{aligned}
$$

where $\omega, \Omega_{c, s}$ and $\omega_{p, s}$ are respectively the RF source, the cyclotron and the plasma angular frequencies of the species $s$.

2.3.2. Grill description The transverse (perpendicular to the $x$ direction) electromagnetic field $\mathbf{E}_{t, \text { grill }}, \mathbf{H}_{t, \text { grill }}$ at the end of the output waveguides, i.e. in the $x=0$ grill plane, can be expanded as a series of excited modes:

$$
\begin{aligned}
& \mathbf{E}_{t, \text { grill }}(x=0, y, z)=\sum_{n=1}^{N} \sqrt{Z_{n}}\left(a_{n}+b_{n}\right) \mathbf{e}_{t, n}(y, z) \\
& \mathbf{H}_{t, \text { grill }}(x=0, y, z)=\sum_{n=1}^{N} \frac{1}{\sqrt{Z_{n}}}\left(a_{n}-b_{n}\right) \mathbf{h}_{t, n}(y, z)
\end{aligned}
$$

where $\mathbf{e}_{t, n}, \mathbf{h}_{t, n}$ are the TE or TM modal functions (explicitly given in reference. [20]) associated to the port $n$ and $Z_{n}$ the impedance of the port $n$ for the current mode. The coefficients $a_{n}$ and $b_{n}$ are the incident and reflected power waves associated to the port $n$. We recall that $N=N_{\text {wg }} \times N_{\text {modes }}$ is the total number of ports, $N_{\mathrm{wg}}$ being the total number of waveguides and $N_{\text {modes }}$ the number of modes in each waveguide. Since the analytical expression of the modes function $\mathbf{e}_{t, n}, \mathbf{h}_{t, n}$ are known, the transverse fields can be analytically expressed in the spectral domain by Fourier transform:

$$
\begin{aligned}
& \tilde{\mathbf{E}}_{t, \text { grill }}\left(n_{y}, n_{z}\right)=\sum_{n=1}^{N} \sqrt{Z_{n}}\left(a_{n}+b_{n}\right) \tilde{\mathbf{e}}_{t, n}\left(n_{y}, n_{z}\right) \\
& \tilde{\mathbf{H}}_{t, \text { grill }}\left(n_{y}, n_{z}\right)=\sum_{n=1}^{N} \frac{1}{\sqrt{Z_{n}}}\left(a_{n}-b_{n}\right) \tilde{\mathbf{h}}_{t, n}\left(n_{y}, n_{z}\right)
\end{aligned}
$$

where $\tilde{\mathbf{e}}_{t, n}$ and $\tilde{\mathbf{h}}_{t, n}$ are the Fourier transform of the modal functions $\mathbf{e}_{t, n}, \mathbf{h}_{t, n}$ and $n_{y}=k_{y} / k_{0}$ and $n_{z}=k_{z} / k_{0}$ are the refractive indexes in $y$ and $z$ directions respectively. These latter expressions will be used for the coupling calculation in the next section. 
2.3.3. Coupling from grill to plasma Following the classical linear-coupling regime [3, 4], the characterization of the plasma medium can be reduced to a spectral surface admittance $\mathbb{Y}_{S}$ defined on the plane that separates the grill from the plasma region:

$$
\tilde{\mathbf{H}}_{t, \text { plasma }}\left(n_{y}, n_{z}\right)=Y_{0} \mathbb{Y}_{S}\left(n_{y}, n_{z}\right) \tilde{\mathbf{E}}_{t, \text { plasma }}\left(n_{y}, n_{z}\right)
$$

where $\tilde{\mathbf{H}}_{t \text {,plasma }}\left(n_{y}, n_{z}\right)$ and $\tilde{\mathbf{E}}_{t, \text { plasma }}\left(n_{y}, n_{z}\right)$ are the Fourier transforms of the transverse magnetic $\mathbf{H}_{t, \text { plasma }}(x=0, y, z)$ and electric fields $\mathbf{E}_{t, \text { plasma }}(x=0, y, z) . \quad Y_{0}$ is the vacuum admittance. The plasma surface admittance $\mathbb{Y}_{s}$, which is discussed in Sec.2.3.5, is generally a $2 \times 2$ complex matrix.

The waveguides of the grill are supposed to be opened through a perfect metallic surface of infinite extent. Due to the continuity of the transverse electric field in the waveguide openings and no tangent electric field on the perfect metallic surface, the transverse magnetic field $\tilde{\mathbf{H}}_{t, \text { plasma }}$ can be expressed using the surface admittance $\mathbb{Y}_{s}$ defined in (10) and the expansion of the electric

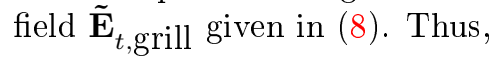

$$
\tilde{\mathbf{H}}_{t, \text { plasma }}\left(n_{y}, n_{z}\right)=\sum_{n=1}^{N} \sqrt{Z_{n}}\left(a_{n}+b_{n}\right) Y_{0} \mathbb{Y}_{s}\left(n_{y}, n_{z}\right) \tilde{\mathbf{e}}_{t, n}\left(n_{y}, n_{z}\right)
$$

Let $\tilde{\mathbf{H}}_{t, \text { metal }}\left(n_{y}, n_{z}\right)$ be the transverse magnetic field related to the current induced on the perfect metallic surface of infinite extent. Due to the continuity between transverse magnetic field in the waveguide openings, one finds from the expansion of the magnetic field in the grill (9) and in the plasma (11) the following equality:

$$
\begin{aligned}
\sum_{n=1}^{N} \frac{1}{\sqrt{Z_{n}}}\left(a_{n}-b_{n}\right) \tilde{\mathbf{h}}_{t, n}\left(n_{y}, n_{z}\right)+ & \tilde{\mathbf{H}}_{t, \text { metal }}\left(n_{y}, n_{z}\right)= \\
& \sum_{n=1}^{N} \sqrt{Z_{n}}\left(a_{n}+b_{n}\right) Y_{0} \mathbb{Y}_{s}\left(n_{y}, n_{z}\right) \tilde{\mathbf{e}}_{t, n}\left(n_{y}, n_{z}\right)
\end{aligned}
$$

The mode matching method is then applied [28]: a linear system is obtained by dot product multiplying both sides of (12) with $\tilde{\mathbf{h}}_{t, m}^{*}\left(n_{y}, n_{z}\right)$ for $m=1, \ldots, N$ and integrating over the spectral domain $\left\{n_{y}, n_{z}\right\}$ of infinite extent. Moreover, by multiplying both sides by $k_{0}^{2} / 4 \pi^{2}$, the left hand side of the equation can be transposed in the spatial domain $\{y, z\}$ thanks to Parseval's theorem:

$$
\begin{aligned}
& \sum_{n=1}^{N} \frac{1}{\sqrt{Z_{n}}}\left(a_{n}-b_{n}\right)\left[\iint_{-\infty}^{+\infty} \mathbf{h}_{t, m}^{*}(y, z) \cdot \mathbf{h}_{t, n}(y, z) d y d z\right. \\
& \left.+\iint_{-\infty}^{+\infty} \mathbf{h}_{t, m}^{*}(y, z) \cdot \mathbf{H}_{t, \text { metal }}(y, z) d y d z\right]= \\
& \sum_{n=1}^{N} \sqrt{Z_{n}}\left(a_{n}+b_{n}\right) C_{m n}
\end{aligned}
$$

where the coupling admittance term $C_{m n}$ is given by:

$$
C_{m n}=Y_{0} \frac{k_{0}^{2}}{4 \pi^{2}} \iint_{-\infty}^{+\infty} \tilde{\mathbf{h}}_{t, m}^{*}\left(n_{y}, n_{z}\right) \mathbb{Y}_{s}\left(n_{y}, n_{z}\right) \tilde{\mathbf{e}}_{t, n}\left(n_{y}, n_{z}\right) d n_{y} d n_{z}
$$


Since the modal function $\mathbf{h}_{t, m}$ is zero on a perfect metallic plane, the term involving $\mathbf{H}_{t, \text { metal }}$ cancels. The orthonormal properties of the modal eigenfunctions[29, 20] leads to:

$$
\frac{1}{\sqrt{Z_{m}}}\left(a_{m}-b_{m}\right)=\sum_{n=1}^{N} \sqrt{Z_{n}}\left(a_{n}+b_{n}\right) C_{m n} \quad \forall m=1, \ldots, N
$$

The linear system of equation.(15) can be rewritten using a matrix formalism:

$$
\sqrt{\mathbb{Z}}^{-1}(\mathbf{a}-\mathbf{b})=\mathbb{C} \sqrt{\mathbb{Z}}(\mathbf{a}+\mathbf{b})
$$

where $\sqrt{\mathbb{Z}}$ is diagonal matrix with $[\sqrt{\mathbb{Z}}]_{i i}=\sqrt{Z_{i}}$ and $\mathbb{C}$ is the coupling admittance matrix with $[\mathbb{C}]_{i j}=C_{i j}$. $\mathbf{a}$ and $\mathbf{b}$ are respectively the incident and reflected waves vectors associated to the port $i$, such as $[\mathbf{a}]_{i}=a_{i}$ and $[\mathbf{b}]_{i}=b_{i}$. The scattering matrix $\mathbb{S}_{\text {grill } / \text { plasma }}$ discussed in Section 2.1 is defined by

$$
\mathbf{b}=\mathbb{S}_{\text {grill } / \text { plasma }} \mathbf{a}
$$

Finally, using (16), one finds:

$$
\mathbb{S}_{\text {grill } / \text { plasma }}=(\mathbb{I}+\sqrt{\mathbb{Z}} \mathbb{C} \sqrt{\mathbb{Z}})^{-1}(\mathbb{I}-\sqrt{\mathbb{Z}} \mathbb{C} \sqrt{\mathbb{Z}})
$$

where $\mathbb{I}$ is the unit matrix.

2.3.4. Antenna power spectrum An important feature of LH antennas is the radiated power spectral density $d p$ that can be computed from the Poynting vector:

$$
d p\left(n_{y}, n_{z}\right)=\Re\left\{\frac{k_{0}^{2}}{4 \pi^{2}}\left[\tilde{\mathbf{E}}\left(n_{y}, n_{z}\right) \times \tilde{\mathbf{H}}^{*}\left(n_{y}, n_{z}\right) \cdot \widehat{x}\right]\right\}
$$

According to previous equations, this power density can be evaluated once the power waves $\mathbf{a}, \mathbf{b}$ for each waveguides have been calculated. In order to compare 1D and 2D modeling, it is possible to define a $1 \mathrm{D}$ spectrum $d p_{z}$ that integrates the contribution of all the $n_{y}$ for a given $n_{z}$ :

$$
d p_{z}\left(n_{z}\right)=\int_{-\infty}^{+\infty} d p\left(n_{y}, n_{z}\right) d n_{y}
$$

Finally, the power conservation must imply that, neglecting the RF losses in the antenna:

$$
\iint_{-\infty}^{+\infty} d p\left(n_{y}, n_{z}\right) d n_{y} d n_{z}=\sum_{n=1}^{N \text { module }} p_{n, \text { in }}\left(1-R C_{n}\right)
$$

where $p_{n \text { in }}$ and $R C_{n}$ are respectively the power incoming from an RF source and the Reflection Coefficient for the n-th module. 
2.3.5. Plasma surface admittance The plasma surface admittance defined in equation.(10) can be evaluated either numerically or analytically depending on the hypothesis made on the wave propagation plasma and on the density profile[3, 4]. In ALOHA, two kinds of wave propagation models have been implemented.

In the first one, the so-called ALOHA-2D mode $-2 \mathrm{D}$ because the plasma parameters depend on two coordinates, namely $n_{y}$ and $n_{z}$ - waveguides cross-section are considered finite in both dimensions and both TE and TM modes are taken into account. In this case, the plasma admittance matrix can either be evaluated numerically [7] or analytically for linear density evolution in terms of Airy and Whittaker functions[3,4]. A brief derivation of the 2D admittance matrix is given in Appendix A. A numerical evaluation of the $2 \mathrm{D}$ admittance is also implemented in ALOHA using the finite element method. The radial domain $x$ is discretized in sub-domains where the field is expanded on second-order polynomials. Using the Galerkin method and setting a WKB condition at the end of the domain, an algebraic system is obtained and solved using a Gaussian elimination.

In the second model, the so-called ALOHA-1D mode, the fast wave coupling is neglected and the waveguide height is considered to be infinite in the poloidal $y$-direction (i.e. $n_{y}=0$ ). In this case, only the TEM and TM modes are excited and the plasma admittance reduces to a scalar that can be expressed analytically in terms of Airy functions for step or linear electron density profiles[12]. A brief derivation of its expression is also given in Appendix A.

A priori, the 1D description of the plasma should not match with the description of the modules since the waveguides have 2D cross-section. However, the scattering matrix formalism characterizes RF structures in terms of incident and reflected power waves in a port and not in terms of electric or magnetic fields. Incident and reflected power waves are only described by the electromagnetic power they carry and a phase, normalized to a port impedance[19]. Thus, the network concept presented in section 2.1 allows to combine ports with different geometries. In ALOHA, rectangular waveguide modes are characterized by their transverse components both in $y$ and $z$ directions. Since in parallel plate waveguides, there is only a transverse component in the $z$ direction, ALOHA-1D only considers the contribution in this direction. The modal impedance, which describes the relationship between transverse components of the electric and magnetic fields, is always the one of the rectangular waveguide, even in $1 \mathrm{D}$ calculation. When one wants to compare scattering parameters with a completely 1D code such as SWAN, then an impedance renormalization is required, cf. Sec.3.3.

The 1D approach is of great interest in spite of the approximations made. Indeed, since the waveguides of the grill are modeled by parallel plate waveguides, two different poloidal lines of waveguides are not coupled by the plasma, which means that there is no coupling between ports associated to those waveguides in the matrix $\mathbb{S}_{\text {grill/plasma }}$. When an antenna is composed of several poloidal lines of waveguides, it is then possible to define a density evolution for each line in order to simulate the effect of poloidal inhomogeneities. Finally, an other strong advantage of the 1D approach is to simplify the double integral of equation.(14) to a simple one, reducing dramatically the computation time while keeping a good agreement with experiments as it will be seen in Sec.3.

2.3.6. Several layers model In a tokamak plasma, the density profile of the scrape-off layer in which the LH antenna radiates may be perturbed by the antenna side limiters or other protruding objects such as other antennas. Thus, the modeling of the electron density by a single linear profile is not deeply realistic and experiments shows that the density decay length - which can be approximated in a first order by $\lambda=n_{e} / \nabla n_{e}$ [30] - is millimetric in front of the grill and centimetric further[31]. Consequently, it seems natural to describe the electron density profile using several layers with different values of $\nabla n_{e}$. 


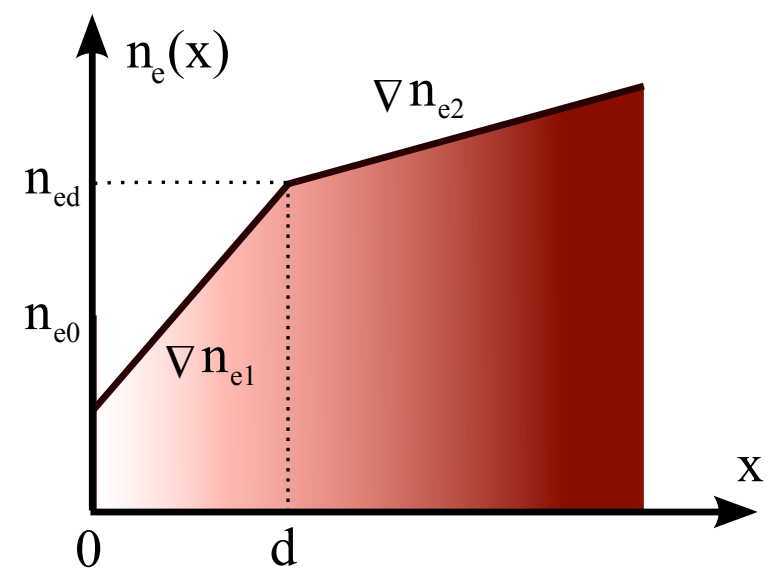

Figure 3. Description of the electronic density profile by two linear profiles in front of an antenna. $x=0$ coincides with the position of the mouth of the grill.

In ALOHA, it is possible to model the plasma density profile by one or two density gradients. The first layer is characterized by a density gradient $\nabla n_{e 1}$ and a thickness $d$; the second layer is characterized by a density gradient $\nabla n_{e 2}$ of infinite extent, as illustrated in figure 3:

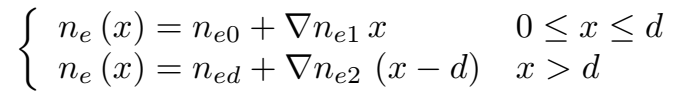

where $n_{e d}=n_{e 0}+\nabla n_{e 1} d$.

Inside the second layer, the solution of the wave equation (1) for $\tilde{E}_{z}$ is similar to the one found in the case of a single layer since the physical requirement as $x \rightarrow+\infty$ is the same (see Appendix A). Inside the first layer, the solution for $\tilde{E}_{z}$ is a combination of Airy and Whittaker functions. The relationship between the both solutions can be found considering that the solutions for the transverse electric and magnetic fields at the interface of both layers $x=d$ have to be continuous and the plasma admittance at the mouth of the antenna can be expressed analytically. Details of the derivation for a $1 \mathrm{D}$ case can be found in Appendix B.

2.3.7. Numerical considerations The results of the coupling calculation described in the previous sections depend on the total number of modes $N_{\text {modes }}$. In order to determine the minimal number of modes to take into account in order to insure the convergence of ALOHA, the total number of modes has been varied and the results on the C3 antenna compared. This is illustrated in figure 4, where the average reflection coefficient and the average electric field at the grill mouth are plotted versus the electron edge density $n_{e 0}$ for the $\mathrm{C} 3$ antenna. When three or more modes are used, the results get very close, indicating that the convergence has been reached.

In figure 5 , the computation time has been plotted versus the total number of modes $N_{\text {modes }}$ for a simple antenna made of 8 independently fed waveguides and for the $\mathrm{C} 3$ antenna ( 57 waveguides per row). As illustrated in the figure 5 , the time complexity of the coupling calculation is function of the number of waveguides and the number of modes taken into account. In both examples, the running time is proportional to the square of the product of the number of waveguides and the 

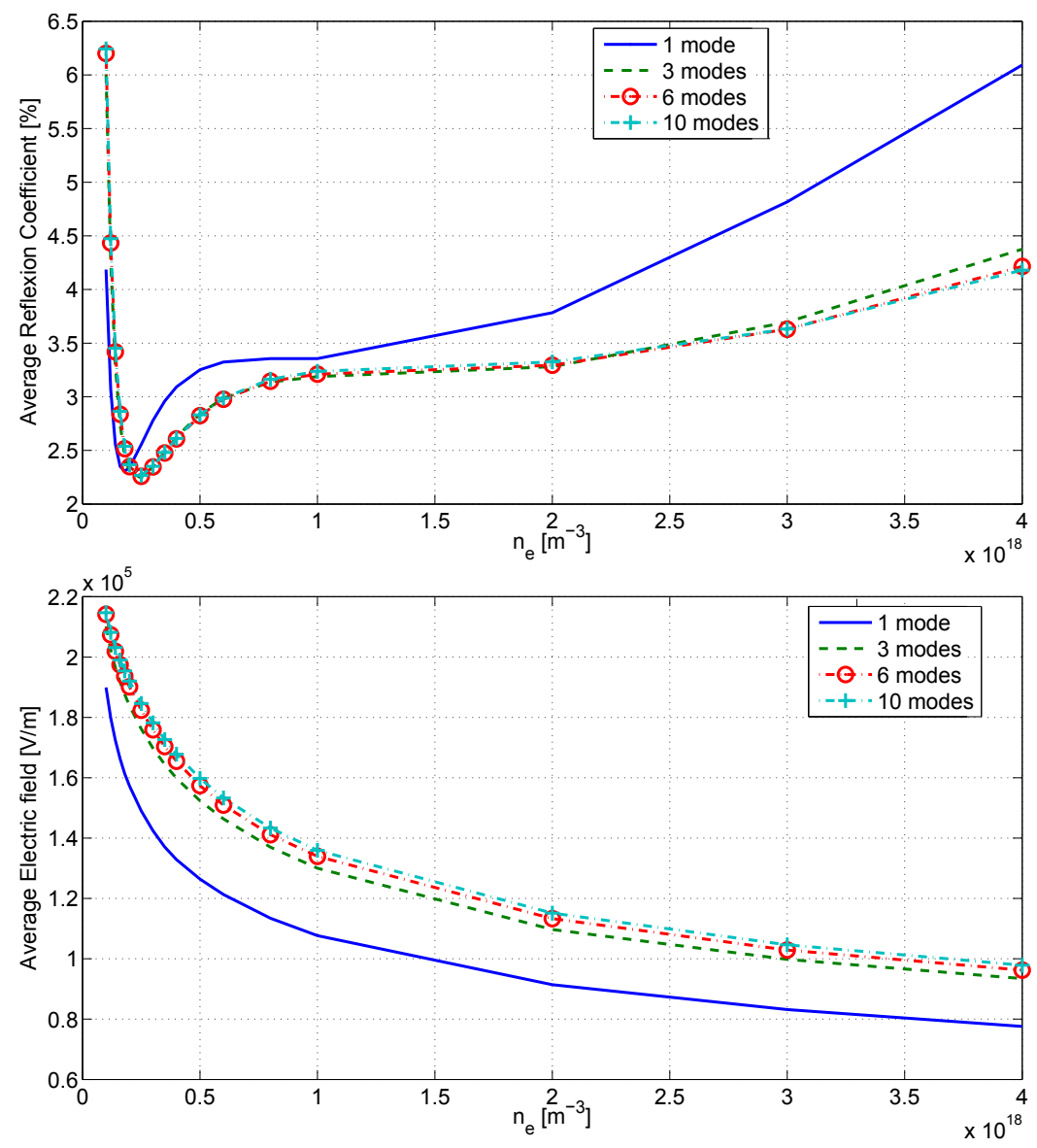

Figure 4. Reflection coefficient (upper) and average electric field at the mouth (lower) vs edge electron density for the C3 antenna calculated by ALOHA, for different total number of modes taken in account.

number of modes, i.e. $O\left(N_{\mathrm{wg}}^{2} \times N_{\text {modes }}^{2}\right)$. In the following of this paper, 3 modes were used for all the simulations made with ALOHA. 3 modes are sufficient at low density $\left(1.10^{17} \sim 20.10^{17} \mathrm{~m}^{-3}\right)$ which is the density range of interest for this work.

When the antenna is large - that is to say in practical terms when there are more than about thirty waveguides -, the numerical computation of the coupling for a $2 \mathrm{D}$ plasma description becomes very difficult. In order to reach the convergence, the precision required on the calculation of the coupling terms in (14) leads to a drastic increase of the computational time. To avoid this problem, a solution consists in introducing losses in the vacuum permittivity. This hypothesis changes the diagonal terms of the dielectric tensor $-S^{\prime}=S-j \delta$ and $P^{\prime}=P-j \delta$ where $\delta$ is a factor of losses, $\delta \ll 1$ and $\delta>0-$ and this eliminates the singularities that appear in the expression of the admittance surface given in Appendix A. However, the implementation of the analytical solution when losses are introduced is very difficult because of the complex arguments that appear in Whittaker functions. It is then more convenient to numerically solve the differential 


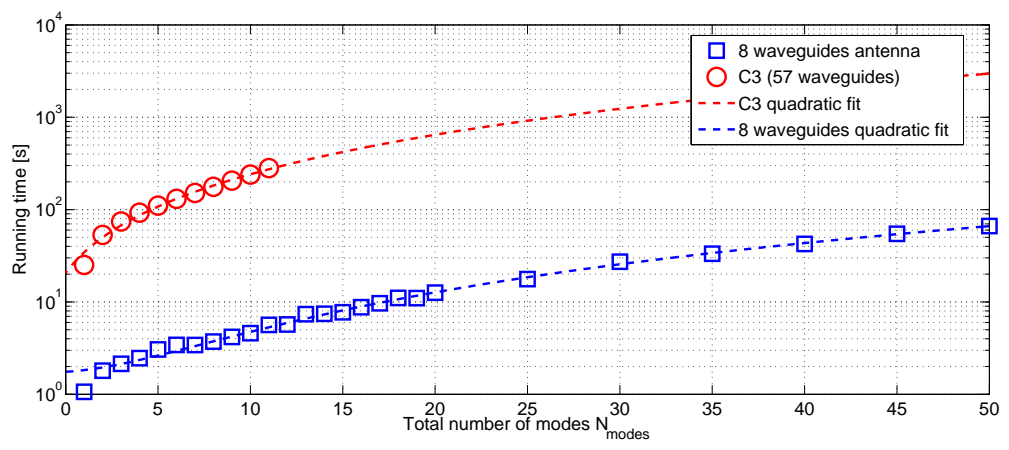

Figure 5. Computation time versus total number of modes for a simple antenna made of 8 waveguides and for the C3 antenna. The dashed lines are quadratic fit in $N_{\text {modes }}^{2}$.

wave equations by using the finite element method as explained in Sec.2.3.5. For the sake of illustration, setting $\delta=10^{-2}$ enables to reduce the computational time by more than two order of magnitude without disturbing the antenna response .

\section{ALOHA validation on Tore Supra antennas}

\subsection{Description of the Tore Supra antennas}

In Tore Supra, the microwave power is generated at $3.7 \mathrm{GHz}$ and launched around $n_{\|}=2$. During the passed two decades, two generations of antennas - referred as C2 [32] and C3 [33] - have been manufactured to radiate more and more power; this trend has resulted in an increasing complexity of RF structures. The $\mathrm{C} 2$ antenna is composed of 16 modules of 2 poloidal $\times 4$ toroidal waveguides while $\mathrm{C} 3$ is made of 16 modules of 3 poloidal $\times 6$ toroidal waveguides separated by 1 passive waveguide. Each module in the $\mathrm{C} 2$ and $\mathrm{C} 3$ antennas has been designed to equally share the incident power coming from a klystron into 8 and 18 active waveguides respectively. Multijunctions are used with mechanical phase shifters in order to create a phase shift of $\pi / 2$ between two successive waveguides on a toroidal line. This nominal phase shift produces a peak parallel index of $n_{\|, 0}=1.82$ for $\mathrm{C} 2$ and $n_{\|, 0}=2.02$ for C3. The ALOHA description of the $\mathrm{C} 2$ and $\mathrm{C} 3$ front faces as view from the plasma is illustrated in figure 6. The C2 antenna, installed in 1991, was removed in 2009 and replaced by the new Passive-Active Multijunction (PAM) antenna[34, 35].

The figure 7 shows the radiated spectral power density of the $\mathrm{C} 2$ and $\mathrm{C} 3$ antennas (in $\log$ scale) calculated by ALOHA-2D when $n_{e 0}$ is close to the cut-off density $\left(1.7 \times 10^{17} \mathrm{~m}^{-3}\right.$ at $\left.3.7 \mathrm{GHz}\right)$. The input power is $2.066 \mathrm{MW}$ for $\mathrm{C} 2$ and $4.032 \mathrm{MW}$ for $\mathrm{C} 3$, corresponding to a power density of $25 \mathrm{MW} / \mathrm{m}^{2}$ at the mouth of the antennas. The main peaks are observed at $n_{y}=0, n_{z}=1.8$ for $\mathrm{C} 2$, and $n_{y}= \pm 0.49 n_{z}=2$ for $\mathrm{C} 3$. These values are coherent with these antennas design values[21, 33].

The spectrum in the $n_{y}$ direction depends on the poloidal power divider of each antenna, which split the power into two or three rows of waveguides for $\mathrm{C} 2$ and $\mathrm{C} 3$ respectively. In the case of the $\mathrm{C} 2$ antenna (left figure 7 ), the spectrum is symmetrical to the $n_{y}=0$ plane because both rows are in phase. In the case of the $\mathrm{C} 3$ antenna (right figure 7), one can observe that the main peaks are located on two opposite poloidal indexes. This result is due to the $\mathrm{TE}_{10}-\mathrm{TE}_{30}$ mode converter, which feeds the three rows of the multijunction and leads to a $\pi$ phase shift between the rows of the 

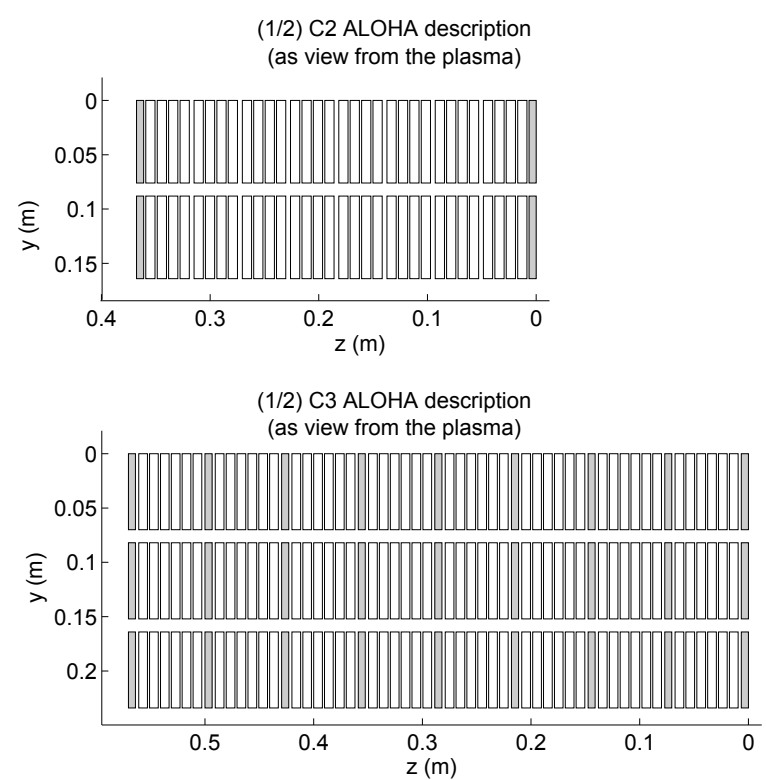

Figure 6. ALOHA description of the upper parts of Tore Supra C2 (top) and C3 LH antennas (bottom). Grey rectangles symbolize passive waveguides. Scale is conserved between both figures.

poloidal junction. This phase generates a poloidal shift of $n_{y}= \pm \frac{\pi}{k_{0} \Delta}= \pm 0.49$ in the $2 \mathrm{D}$ spectrum, where $\Delta=82 \mathrm{~mm}$ is the distance between two guides in the poloidal direction and $k_{0}$ the vacuum wavenumber at $3.7 \mathrm{GHz}$. This phase shift has not been compensated at the time of the design of the antenna. However, LHCD Tore Supra experiments show that there is no observable difference in the current drive efficiencies between the $\mathrm{C} 2$ (no poloidal $n_{y}$ shift) and $\mathrm{C} 3$ antennas (non zero poloidal $n_{y}$ shift).

The 1D spectrum for both antennas - as defined in equation.(20) - is illustrated in figure 8. Both 1D and 2D modeling give nearly the same 1D spectrum. This result is not necessary in contradiction with the influence of the fast wave coupling: in the usual electron density range in front of the Tore Supra LH antennas, the fast wave is cut-off and then carries only reactive energy while the slow wave propagates, carrying radiated power. Thus, equation.(20) gives nearly the same result since only the real part of the spectrum is considered. This reactive energy appears in the imaginary part of the spectrum that can be build in a similar way as equation.(19) and may contributes to the apparition of second order reflective waves inside the waveguides. 

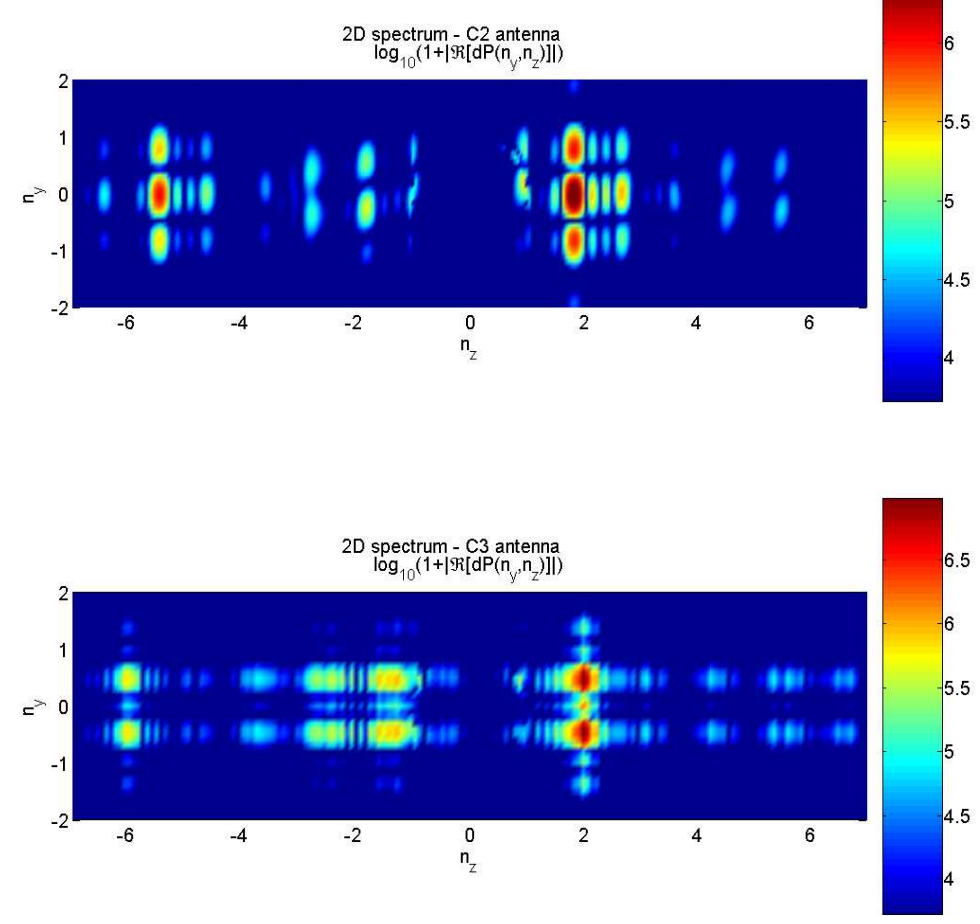

Figure 7. 2D normalized radiated power spectrum of $\mathrm{C} 2$ (upper) and $\mathrm{C} 3$ antennas (lower), calculated using the one layer model with $n_{e 0}=2.0 \times 10^{17} \mathrm{~m}^{-3}, \lambda_{n 1}=2 \mathrm{~cm}$ and $B_{0}=2.95 \mathrm{~T}$.
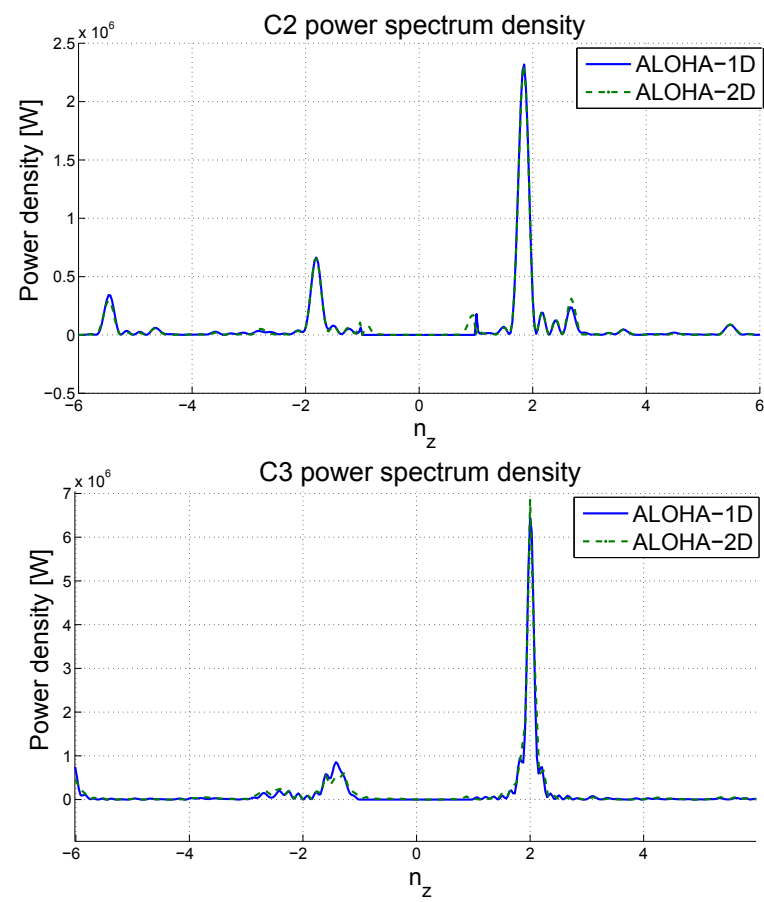

Figure 8. 1D radiated power spectrum calculated by ALOHA-1D and ALOHA-2D of C2 (top) and $\mathrm{C} 3$ antennas (bottom). For both figures, the plasma is described by one layer model with $n_{e 0}=2.0 \times 10^{17} \mathrm{~m}^{-3}, \lambda_{n 1}=2 \mathrm{~cm}$ and $B_{0}=2.95 T$. 


\subsection{Comparison with experimental results}

During October and November 2008, dedicated experiments were carried out in Tore Supra in order to compare the measured power reflection coefficients on the $\mathrm{C} 2$ and $\mathrm{C} 3$ antennas with the numerical results from the ALOHA code. In order to avoid possible non-linear effects[36, 37], low power pulses were used, i.e. pulses for which the power density at the mouth of the antenna is lower than $2 \mathrm{MW} / \mathrm{m}^{2}$ (corresponding to a total requested input power of $100 \mathrm{~kW}$ for $\mathrm{C} 2$ and $75 \mathrm{~kW}$ for C3). The parameters for the pulse \#43016 are shown in figure 9. A large variation of density in front of the antenna between $0.3 \times 10^{17} \mathrm{~m}^{-3}$ and $13 \times 10^{17} \mathrm{~m}^{-3}$ was obtained by varying the distance between the last closed flux surface (LCFS) and the antenna during the pulse (cut-off density at $3.7 \mathrm{GHz}$ is $n_{e c}=1.7 \times 10^{17} \mathrm{~m}^{-3}$ ). Electron density was measured with the Langmuir probes embedded into the LH antennas. The reported experiments were carried out at high magnetic field $\left(B_{0}=3.85 \mathrm{~T}\right.$ at the center of the plasma) and medium density $\left(n_{e}(r / a=0) \leqslant 4,5.10^{19} m^{-3}\right.$ and $\left.n_{e}(r / a=1) \leqslant 1.10^{19} m^{-3}\right)$, so the wave is fully accessible to the confined plasma and the assumption of full absorption of the wave is justified.

TS pulse \#43016
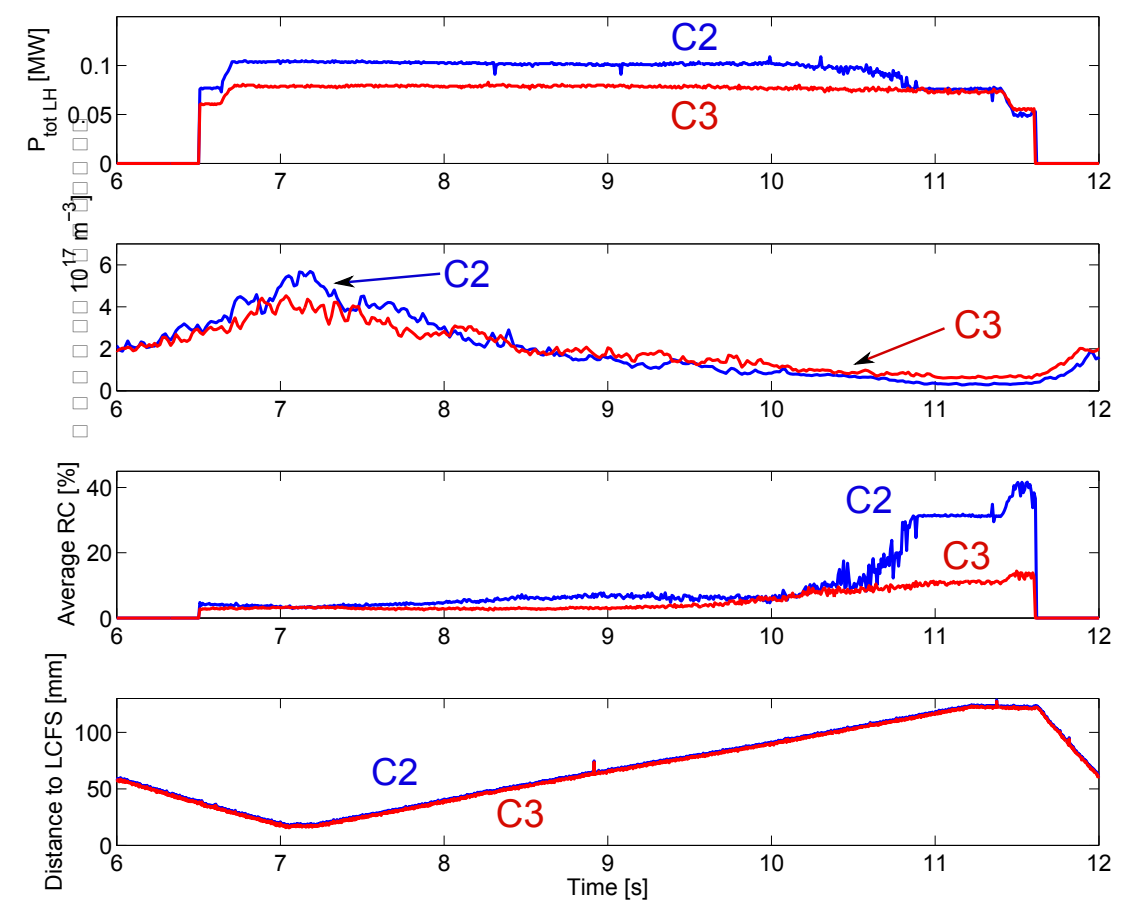

Figure 9. Tore Supra pulse \#43016. Top to bottom: total power $\left(P_{\text {tot }}\right)$; electron density in front measured by Langmuir probes in front of the antenna; average reflection coefficient (RC); distance between the antenna mouths and the plasma LCFS.

In ALOHA, the edge plasma is described with a linear electron density profile and no vacuum layer in front of the grill. Reciprocating probe measurements from a Retarding Field Analyzer 
(RFA) have been used to estimate typical decay length in front of the antennas[38]. The connection lengths $L_{c, L H}$ in front of $\mathrm{C} 2$ and $\mathrm{C} 3$, corresponding to the distance between protruding side limiters, are known to be $40 \mathrm{~cm}$ and $60 \mathrm{~cm}$. The connection length $L_{c, R F A}$ of the RFA probe is evaluated knowing the vacuum vessel and plasma configuration. The scrape-off thickness for a diffusive model in front of the antenna is $\lambda_{n 1, L H}=\frac{n_{e 0}}{\nabla n_{e 1}}=\sqrt{\frac{D_{\perp} L_{c, L H}}{c_{s}}}$. Similarly, the scrape-off thickness measured by the RFA is $\lambda_{n 1, R F A}=\sqrt{\frac{D_{\perp} L_{c, R F A}}{c_{s}}}$. Assuming that the ratio of the cross-field diffusion coefficient $D_{\perp}$ and the plasma acoustic velocity $c_{s}$ is constant in the scrape-off layer, one finds that $\lambda_{n 1, L H}=\sqrt{\frac{L_{c, L H}}{L_{c, R F A}}} \lambda_{n 1, R F A}$.

In figure 10, the experimental reflection coefficients for different electron densities, measured during Tore Supra pulses \#43014-43016 for the upper modules of the C2 antenna are plotted. The density is measured with the nearest Langmuir probe placed at the center of the $\mathrm{C} 2$ antenna. Plain black line corresponds to the reflection coefficient calculated using ALOHA for different electron density values $n_{e 0}$ at the mouth of the antenna and with $\lambda_{n 1}=7 \mathrm{~mm}$. For the C2 antenna, RFA measurements give $\lambda_{n 1}$ in $[3.3,6.5] \mathrm{mm}$, which is in agreement with ALOHA results.

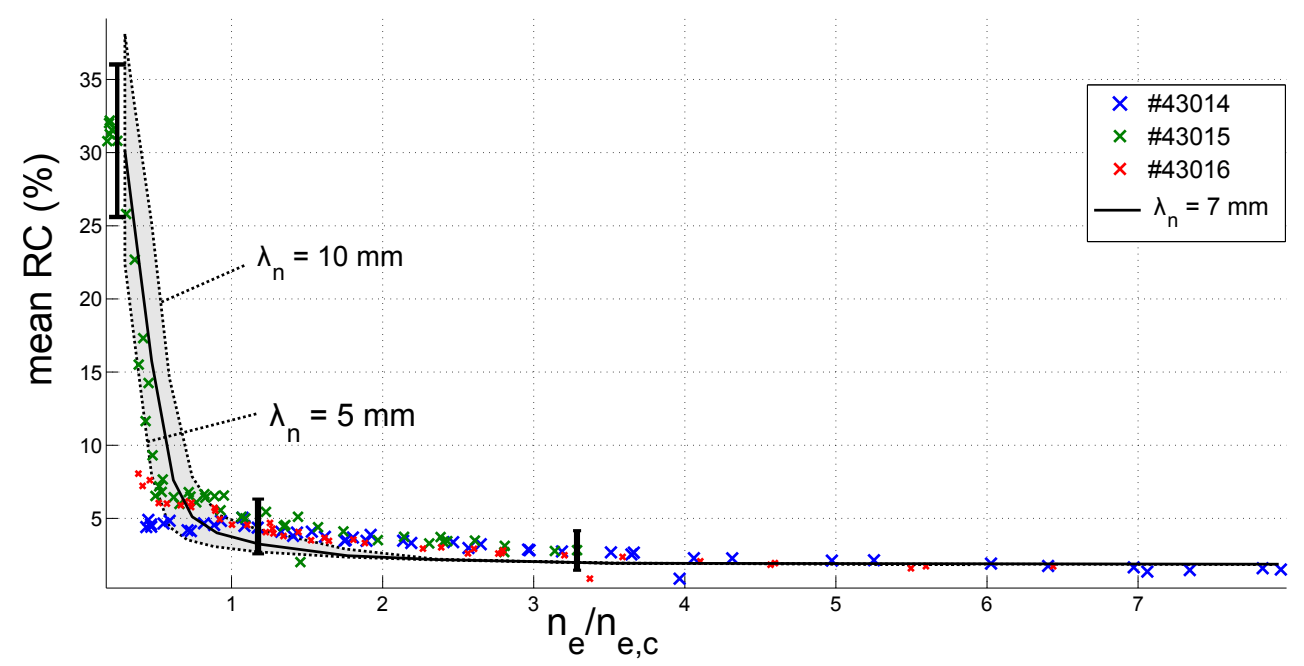

Figure 10. Average reflection coefficient (in percents) versus electron density (normalized to LH cut-off density) for the $\mathrm{C} 2$ antenna. Measured reflection coefficients are taken from Tore Supra pulses number \#43014-43016. Black curve corresponds to a $7 \mathrm{~mm}$ scrape-off thickness $( \pm 3 \mathrm{~mm})$. Dotted lines are for $\lambda_{n 1}=5$ and $10 \mathrm{~mm}$. Three error bars indicating the power measurements uncertainty are illustrated.

In figure 11, the experimental reflection coefficients at different electron densities, measured during Tore Supra pulses \#43014-43016 for the 4 first lower modules of the C3 antenna are plotted (the 4 other modules were not firing for these pulses). The density is measured with the nearest Langmuir probe placed at the bottom of the $\mathrm{C} 3$ antenna. The same method as previously has been used to determine realistic values of the density decay length $\lambda_{n 1}$ in front of the antenna. ALOHA predictions show a good agreement with experimental measurements for all density values.

These dedicated experiments in Tore Supra show that ALOHA results are in good agreement with the experimental measurements made for both multijunction antennas $\mathrm{C} 2$ and $\mathrm{C} 3$. An 


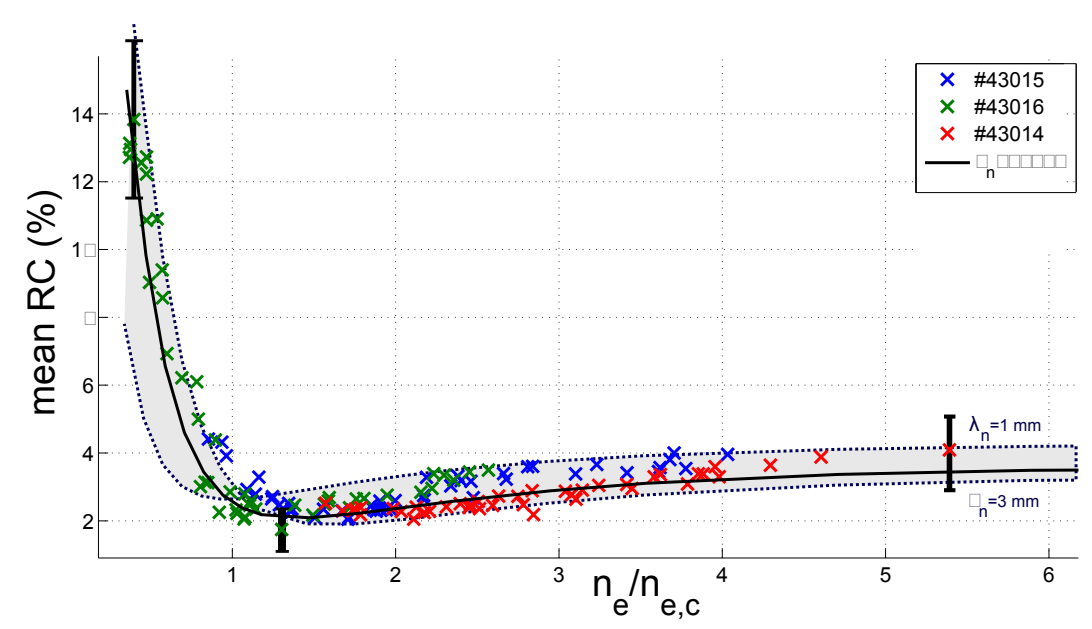

Figure 11. Average reflection coefficient (in percents) versus electron density (normalized to LH cut-off density) for the C3 antenna. Measured reflection coefficients are taken from Tore Supra pulses number \#43014-43016. Black curve corresponds to a $2 \mathrm{~mm}$ scrape-off thickness $( \pm 1 \mathrm{~mm})$.

Three error bars indicating the power measurements uncertainty are illustrated.

extensive comparison between the new PAM antenna which has been installed on Tore Supra in 2009 and ALOHA will be detailed in a future dedicated paper.

\subsection{Comparison of ALOHA and SWAN on C3}

In this section, we compare the results of the coupling code SWAN[39] to ALOHA-1D on a single line of waveguides of the Tore Supra C3 antenna. As described in the previous section, one row of this antenna is made of 8 modules each composed of 6 waveguides. A passive waveguide is inserted between each module and on each side of the antenna, which leads to a total of 57 waveguides as illustrated in figure 6 .

In the SWAN code, waveguides are modeled by parallel plates instead of rectangular waveguides. Thus, the mode impedance is not the same between both codes. In order to compare the calculated grill/plasma scattering parameters we re-normalized the ALOHA mode impedances to the SWAN mode impedances according to reference.[19]. The evolution of the coupling between the first active waveguide of the $\mathrm{C} 3$ antenna with all other waveguides of the row is expressed by the scattering parameters $\left[\mathbb{S}_{\text {grill/plasma }}\right]_{i, 2}$ with $i \in 1 \ldots 57$. Results are illustrated in figure 12 , in which only the coupling between fundamental mode is plotted. The curves for both SWAN and ALOHA match with a good agreement. Some numerical instabilities due to integration routines (cf. right figure 12) have however been experienced in the SWAN code, especially for large antennas: these numerical problems have been fixed in ALOHA. As the coupling coefficients are the same, the antenna spectra for both codes match as well.

\subsection{Comparison of ALOHA and TOPLHA on C2}

Numerical comparisons between ALOHA and TOPLHA[9] on two modules of the Tore-Supra C2 antenna were performed. TOPLHA incorporates the Finite Element Lower Hybrid Solver 

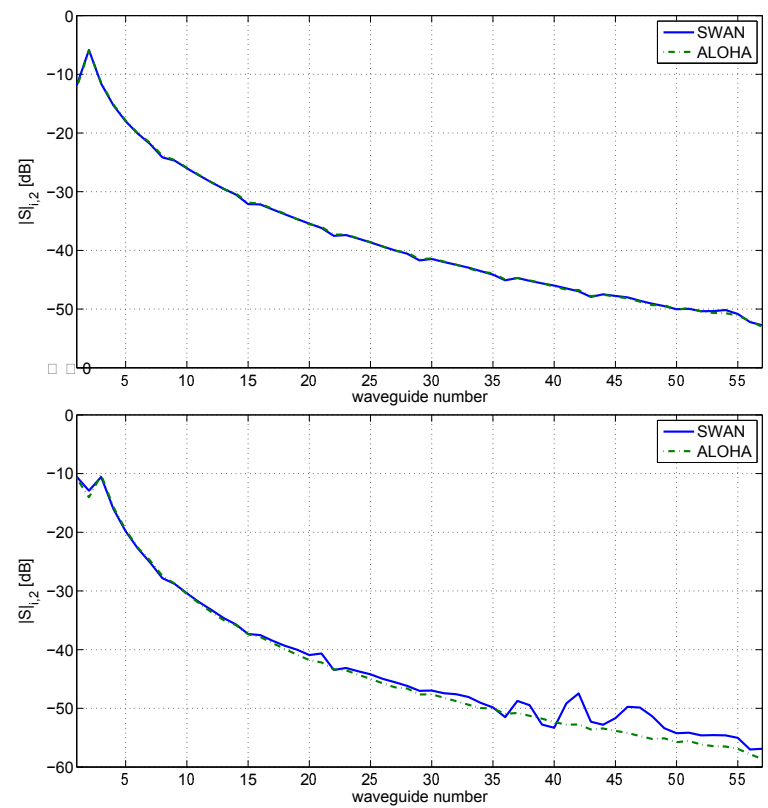

Figure 12. Amplitude (in $\mathrm{dB}$ ) of the scattering parameter $\left[\mathbb{S}_{\text {grill } / \text { plasma }}\right]_{i, 2}$ for the first row of the Tore Supra C3 antenna calculated by the SWAN and the ALOHA codes. Plasma parameters: $n_{e 0}=2.10^{17} \mathrm{~m}^{-3}$ (top) and $n_{e 0}=5.10^{17} \mathrm{~m}^{-3}$ (bottom) $\cdot \lambda_{n 1}=2 \mathrm{~cm}, 3 \operatorname{modes}(1 \mathrm{TEM}+2 \mathrm{TM})$ for both codes.

FELHS[40], and thus intrinsically takes into account fast and slow waves but presents the drawback to involve an intensive computational cost.

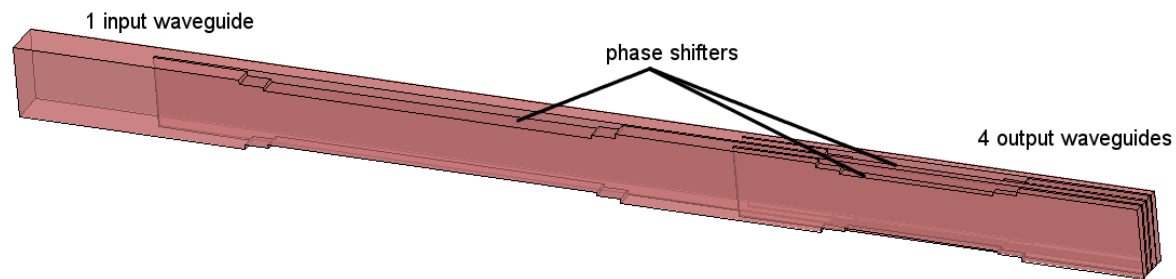

Figure 13. RF model of one module of Tore Supra C2 antenna.The waveguides cross-section dimensions are $76 \mathrm{~mm} \times 8.5 \mathrm{~mm}$.

Comparisons have been made on two side-by-side modules of the $\mathrm{C} 2$ antenna with a linear electron density profile in front of the antenna mouth. The reflection coefficient, i.e. ratio of reflected power to forward power for one module, was calculated for different density $n_{e 0}$ at the mouth of both modules. As seen in figure 14 for $\lambda_{n 1}=2 \mathrm{~cm}$, both codes are in good agreement for both predicted reflected power and launched power spectra.

This benchmark shows that ALOHA-2D and TOPLHA give the same results on a simple multijunction antenna, which gives good confidence in the ALOHA-2D results. Moreover, the 


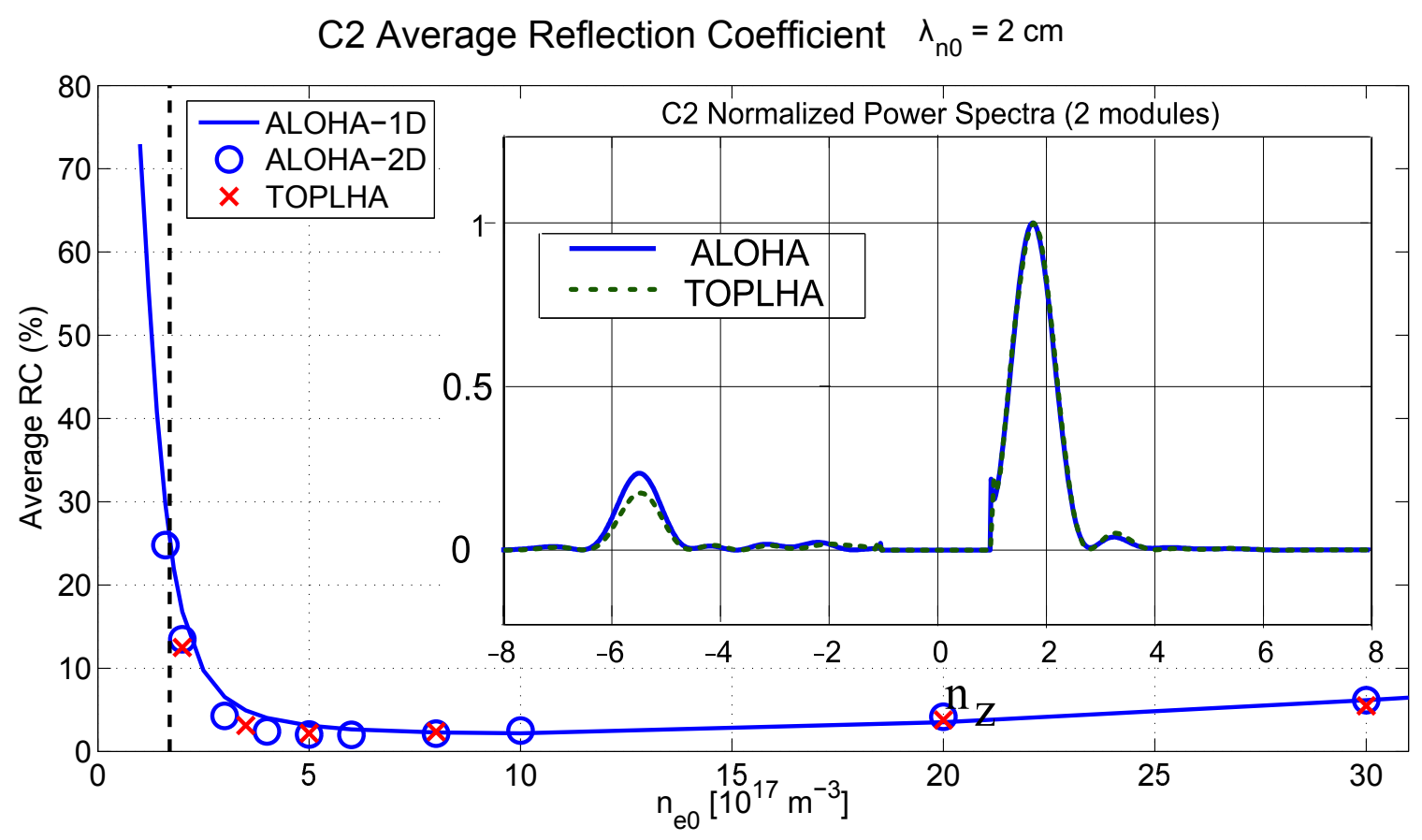

Figure 14. Reflection coefficients as a function of the electron density $n_{e 0}$ in front of two C2 modules and comparison of launched spectrum for initial electron density $n_{e 0}=5 \times 10^{17} \mathrm{~m}^{17}$ and scrape-off layer thickness of $\lambda_{n 1}=2 \mathrm{~cm}$.

ALOHA-1D results agree as well, which means that the $2 \mathrm{D}$ effects are limited on this example. Another comparison between ALOHA and TOPLHA was therefore made on a more complex antenna structure, the ITER LH antenna design cf. Sec.4.2.

\section{Design of future LH antennas}

\subsection{COMPASS}

In the frame of the collaboration between the Prague Institute of Plasma Physics and the CEA/IRFM, two preliminary designs of a $3.7 \mathrm{GHz}$ Lower Hybrid antenna for the COMPASS tokamak were studied. In both designs, the antenna is made of 4 modules, 2 in the poloidal direction and 2 in the toroidal direction, filling the $\mathrm{mm}$ plug dimensions. Two klystrons will feed the two modules, using a poloidal $3 \mathrm{~dB}$ splitter such as an hybrid junction. Both designs differ by the number of output waveguides per module, either 6 or 8 . The output waveguide cross-section is $70 \times 9 \mathrm{~mm}$ for the 6 -waveguides design and $70 \times 7 \mathrm{~mm}$ for the 8 waveguides design. The peak parallel index launched by the structure is either $n_{\|}=2.5$ or 2 respectively. In both designs, the phase shift between waveguide is set to $\pi / 2$ by mechanical phase shifters inside a multijunction. A front and cut view of both kinds of module are shown on figure 15 .

The phase shifters as well as the complete multijunctions dimensions have been modeled and optimized using the HAMAC plug-in of ALOHA. The scattering matrices obtained with HAMAC have been compared to those obtained by the RF software HFSS with an average amplitude error of 

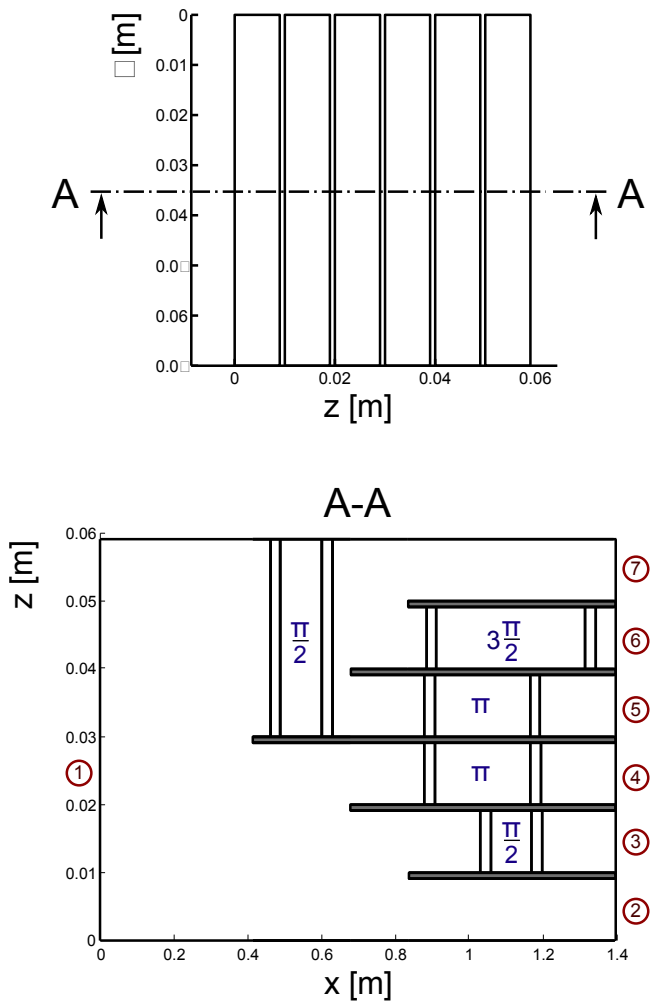

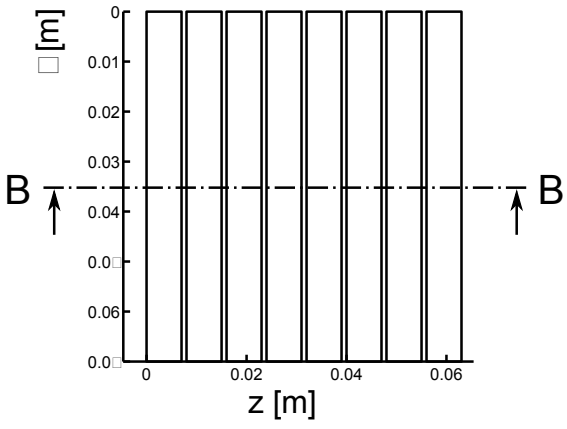

B-B

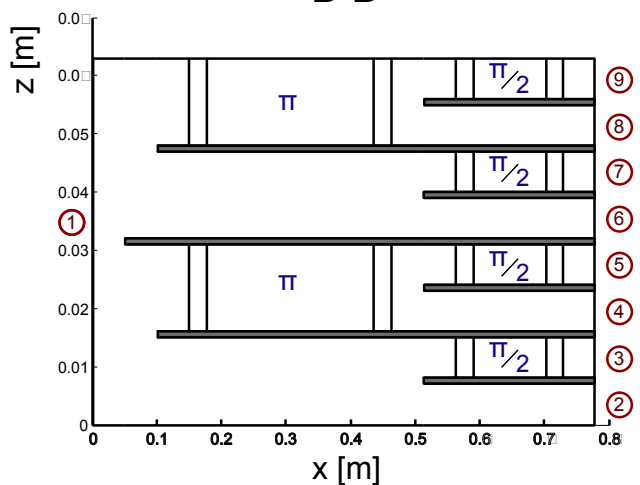

Figure 15. Illustration of the geometry of two kinds of multijunction module designed for a $3.7 \mathrm{GHz}$ LH COMPASS antenna. Left figure: 6 waveguides design. Right figures: 8 waveguides design. Top figures: module front-face. Bottom figures: module cut view (not equally scaled). The RF input is coming from port 1 . Plasma is in front ports $i>1$. Phase shifters locations and their phase shift values are given.

order $10^{-2}$ and $0.5^{\circ}$ average phase error. It is to be noted that the error depends on both HAMAC and HFSS requested precision. The precision as well as the computation time in HAMAC is set by the number of modes (TE and TM) taken into account, whereas in HFSS it depends on the mesh precision and on the requested maximum difference in the magnitude of the scattering parameters between two consecutive calculations. A few number of modes is necessary to converge to a working solution and leads to fast evaluation of the scattering matrix. Thus, HAMAC is well adapted to antenna optimizations, a task which may require a lot of different calculations.

Once the scattering matrix of the modules calculated, they are integrated into ALOHA in order to evaluate the coupling properties of the complete antenna. In figure 16, the average reflection coefficient and the radiated spectrum for a row of two module is plotted for each design (12 or 16 waveguides). In order to compare the accuracy of the HAMAC plug-in, the scattering matrices of the modules have been calculated by both HAMAC and HFSS for each design and thus integrated in ALOHA. Reflection coefficients, which are heavily dependent of the RF structure, are in good agreement for both designs. The power spectrum, less dependent of the RF structure than on the phasing between waveguides, also perfectly match. These results demonstrate how convenient and efficient the HAMAC plug-in is to calculate LH multijunction scattering matrix in a preliminary 
design.
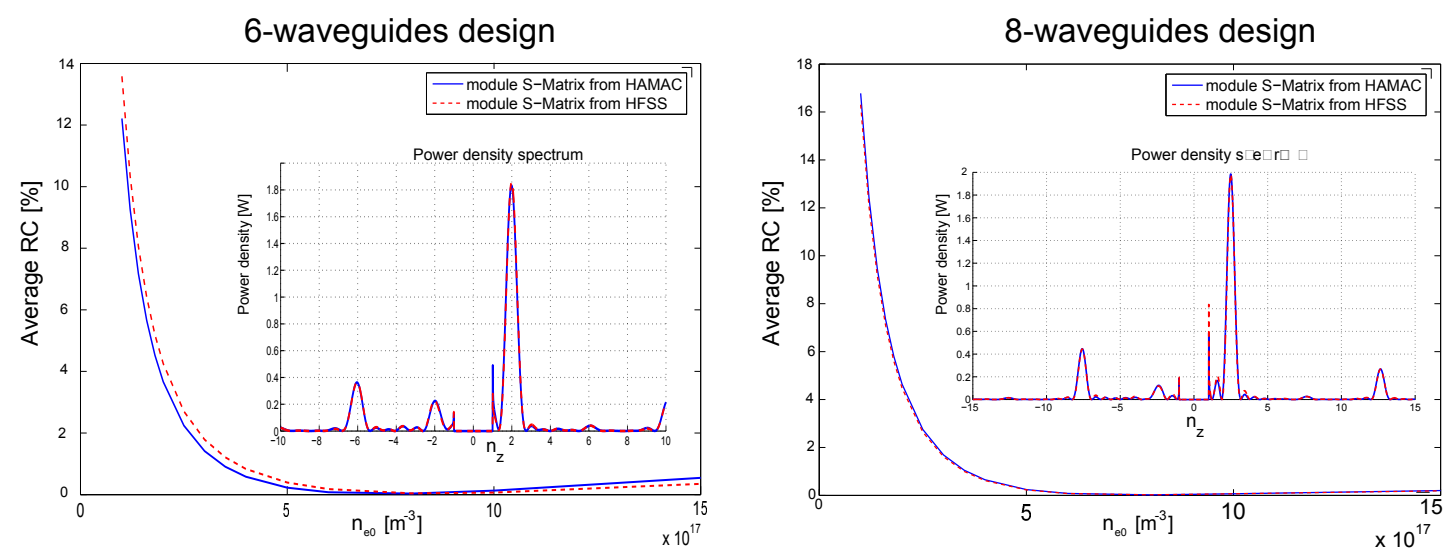

Figure 16. Average reflection coefficient and radiated power spectrum for both kinds of COMPASS 3.7 GHz LH antenna design. Left: 6-waveguides design; Right: 8-waveguides design. For all figures, the ALOHA calculations have been made with the module scattering matrix calculated by HAMAC and HFSS.

\subsection{ITER relevant PAM}

Following the ITER STAC recommendation, an EFDA task has been created in order to initiate the conceptual design, R\&D, procurement and installation of a LHCD system on ITER. The EFDA task HCD-08-03-01 has reported a revised $5 \mathrm{GHz}$ Lower Hybrid system being able to deliver $20 \mathrm{MW} \mathrm{CW}$ on ITER and sustain the expected high heat flux [2]. This work has been achieved in collaboration with ITER organization under worldwide contributions from China, India, Korea and USA on top of EFDA. The LH antenna design presently foreseen for ITER is a Passive-Active Multijunction (PAM) concept, which has been successfully validated on FTU[41] and more recently on Tore Supra in steady-state conditions[34, 35].

In the frame of this EFDA task, a benchmark of the LH coupling codes TOPLHA and ALOHA has been conducted[42, 43]. Comparison between both codes were made on one part of a $5 \mathrm{GHz}$ module designed to launch a power spectrum centered around $n_{\|}=2.0$, as described in reference.[44]. This module consists in 8 active waveguides, corresponding to the $1 / 144$ th of the total antenna. The RF model of a module has been built on HFSS in order to deduce the scattering parameters of the antenna. The power is equally divided in narrow waveguides with a low unbalance: the scattering parameters $S_{n 1}$ which give the ratio between incident power waves coming from port 1 (input feeding waveguide) to port $n \in[2,8]$ (active output waveguides) is $\left|S_{n 1}\right|^{2}=0.125 \pm 0.01$, close to the ideal case of $1 / 8 \mathrm{th}$. The phase shifters arrangement is achieved as illustrated in figure 17 . The phase pitch between two active waveguides is $270^{\circ} \pm 5^{\circ}$.

Two kinds of plasma scenario consisting of two different density gradients were bench-marked with ALOHA-1D, ALOHA-2D and TOPLHA. The density decay lengths used were $\lambda_{n 1}=2 \mathrm{~mm}$ and $20 \mathrm{~mm}$. The reflection coefficient for different densities $n_{e 0}$ at the mouth of the module for $\lambda_{n 1}=2 \mathrm{~mm}$ is plotted in figure 18. Some slight differences between ALOHA-1D and ALOHA2D/TOPLHA are observed for high density values. In this range of density, the cross-term in the cold plasma dielectric tensor $(D)$ becomes non-negligible and generates a coupling between fast and 


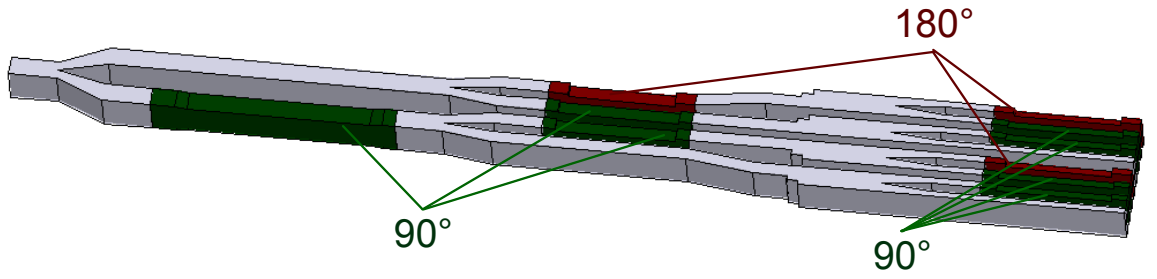

Figure 17. Geometry of the ITER-like PAM multijunction under benchmark. The mechanical phase shifter arrangement is illustrated. The waveguides height is $58 \mathrm{~mm}$, the active and passive waveguide widths are $9.25 \mathrm{~mm}$ and $7.25 \mathrm{~mm}$ respectively. The septum width between waveguides is $3 \mathrm{~mm}$.

slow waves. Therefore, the difference between ALOHA-2D and TOPLHA are smaller, since both codes handle fast and slow waves coupling. Comparisons have also been made on the calculated spectrum and both codes give almost identical results as shown in figure 18. Table 1 reports the directivity of the module, calculated as the ratio of the positive part of the spectrum over the total power, i.e.

$$
\mathcal{D}=\frac{\int_{1}^{+\infty} d p_{z} d n_{z}}{\int_{-\infty}^{+\infty} d p_{z} d n_{z}}
$$

where $d p_{z}$ is defined in equation.(20). Results are in good agreement for all values.
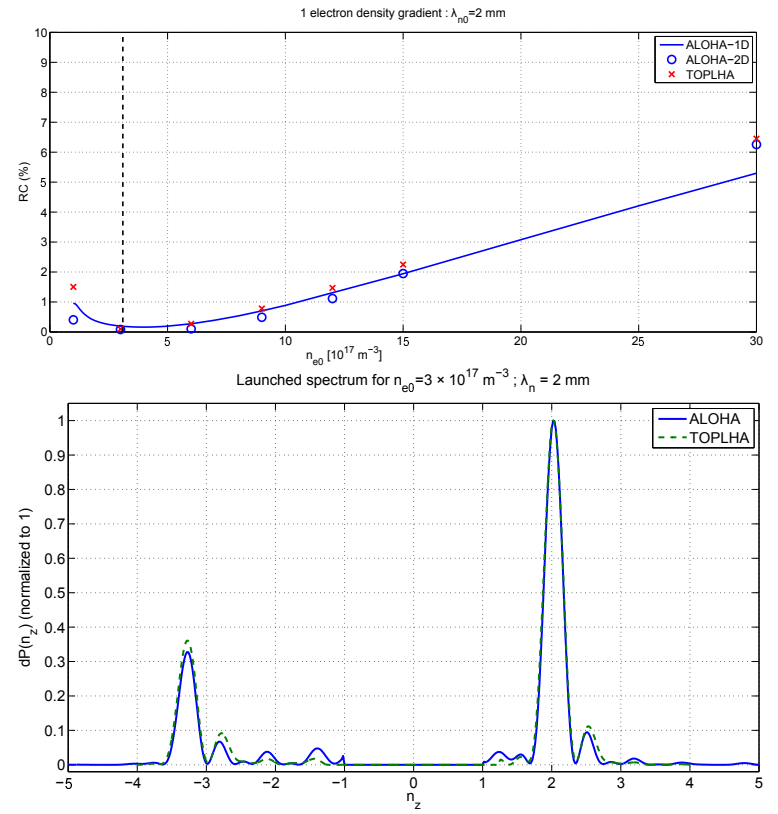

Figure 18. Top: Reflection coefficient vs electron edge density. Bottom: power spectrum density. Linear density evolution characterized by $\lambda_{n 1}=2 \mathrm{~mm}$.

Since ALOHA and TOPLHA do not solve the plasma coupling problem in the same way, the computation time and computational power required are different. For one density point and a 


\begin{tabular}{|c|c|c|c|c|}
\hline $\mathcal{D}$ & \multicolumn{2}{|c|}{ ALOHA } & \multicolumn{2}{c|}{ TOPLHA } \\
\hline$n_{e 0}\left[10^{17} \mathrm{~m}^{-3}\right] \backslash \lambda_{n 1}[\mathrm{~mm}]$ & 2 & 20 & 2 & 20 \\
\hline 3 & $72.2 \%$ & $71.7 \%$ & $70.5 \%$ & $68.9 \%$ \\
\hline 9 & $64.1 \%$ & $65.7 \%$ & $64.4 \%$ & $65.6 \%$ \\
\hline 30 & $59.3 \%$ & $59.25 \%$ & $57.2 \%$ & $59.6 \%$ \\
\hline
\end{tabular}

Table 1. Comparison of directivity $\mathcal{D}(23)$ between TOPLHA and ALOHA for a unique module described in reference.[44].

single module, we report in Table 2 the average computation times for both codes. As said before, 2D calculation in ALOHA increases the computation time in the comparison to the $1 \mathrm{D}$ approach. In TOPLHA, the coupling problem is solved by the FEM method, which in this case requires a larger computational power than a desktop computer in order to get a result in a reasonable time.

\begin{tabular}{|c|c|c|}
\hline Code & Computation time & Computer \\
\hline \hline ALOHA-1D & $<10 \mathrm{~s}$ & Desktop computer (Intel Core@1.86GHz,1GB) \\
\hline ALOHA-2D & $\sim 2 \mathrm{~h}$ & Desktop computer \\
\hline TOPLHA & $\sim 10 \mathrm{~min}$ & Cray XT4 system, $4002.3 \mathrm{GHz} / 8 \mathrm{~GB}$ cores. \\
\hline
\end{tabular}

Table 2. Average computation time and computational power required for the calculation of a density point for a single module.

To conclude this section, results from both codes are in good agreement, update and complete those of reference.[44] on an ITER relevant PAM module. The reflected power of this design does not exceed $10 \%$ even for very high edge electron densities. The similar results obtained by both approach show that ALOHA, especially ALOHA-1D, are fast and reliable tools for LH antenna design.

\section{Conclusion}

The ALOHA code has been developed to model the Lower Hybrid antenna coupling. In this code, multijunction antennas can be described either by the HAMAC mode-matching code or by any full-wave RF software in order to take into account their detailed geometry. The plasma density layers in front of the antenna can be defined by one or two linear models in order to allow a more realistic description of the scrape-off density profile in front of the antenna. The coupling between the plasma and antenna is treated with either fast and slow waves (ALOHA-2D) or slow wave only (ALOHA-1D) via a surface admittance formulation.

Comparisons between Tore Supra LH antennas measurements (collected during dedicated experiments) and ALOHA results show good agreements. The Benchmarks with LH coupling codes SWAN and TOPLHA were also successfully conducted for both ALOHA-1D and ALOHA2D. The ALOHA plug-in HAMAC has already been used in the design of the LH antenna for the COMPASS tokamak and had demonstrate how convenient it was to design then optimize multijunction structures. ALOHA has also been used in the design of the ITER LH antenna showing how the code and its numerical approach was fast and reliable to address such large and complex RF structure.

The ALOHA code is integrated in the framework of the EFDA Integrated Tokamak Modeling Task Force (ITM-TF). More precisely, ALOHA is part of the Integrated Modeling Project 5 
(IMP \#5), a program about "Heating, Current Drive and Fast Particles" aiming to develop a package of codes for the prediction and the interpretation of heating, current drive and fast particle effects. The ITM-TF was set up in 2004 with the long-term objective to provide the EU community with all the integrated codes required for assessing and analyzing future ITER discharges, with the highest degree of flexibility, confidence and reliability[45]. The ALOHA code has been adapted to run on the ITM servers (the Gateway) and made conform to ITM standards, especially in terms of communication via ITM data structures, i.e. Consistent Physical Objects (CPOs)[46]. ALOHA can be used as an actor in the ITM simulation platform (Kepler), which will facilitate benchmarking with other LH coupling codes or integration in more complex work flows, for instance as an input to LH ray-tracing and Fokker-Planck codes.

\section{Acknowledgements}

This work, supported by the European Communities under the contract of Association between EURATOM and CEA, was carried out within the framework of the European Fusion Development Agreement. The views and opinions expressed herein do not necessarily reflect those of the European Commission.

\section{References}

[1] A. Tuccillo et al. Progress in LHCD: a tool for advanced regimes on ITER. Plasma Physics and Controlled Fusion, 47(12B):363-377, 2005.

[2] Gia Tuong Hoang et al. A lower hybrid current drive system for ITER. Nuclear Fusion, 49(7), 2009.

[3] M. Brambilla. Waveguide launching of lower hybrid waves. Nuclear Fusion, 19:1343-1357, October 1979.

[4] A. Bers and K.S. Theilhaber. Three-dimensional theory of waveguide-plasma coupling. Nuclear Fusion, $23(1): 41-48,1983$.

[5] Xavier Litaudon. Etude théorique et expérimentale du couplage de l'onde hybride dans Tore Supra et JET au moyen d'antennes à multijonctions. PhD thesis, Université de Provence, June 1990.

[6] Didier Moreau and T.K. Nguyen. Couplage de l'onde lente au voisinage de la fréquence hybride basse dans les grands tokamaks. Technical report, EUR-CEA-FC1246 Euratom-CEA, 1984.

[7] M.A. Irzak and O.N. Shcherbinin. Theory of waveguide antennas for plasma heatinh and current drive. Nuclear Fusion, 35(11):1341-1356, 1995.

[8] Daniele Milanesio, Vito Lancelloti, Orso Meneghini, Riccardo Maggiora, G. Vecchi, and Roberto Bilato. TOPLHA: an accurate and efficient numerical tool for analysis and design of $\mathrm{lh}$ antennas. In Radio Frequency Power In Plasmas: Proceedings Of The 17th Topical Conference, Aip Conference Proceedings, volume 933, pages 301-304, 2007.

[9] Orso Meneghini, Ron Parker, Julien Hillairet, Marc Goniche, Damien Voyer, and Roberto Bilato. TOPLHA and ALOHA: comparison between lower hybrid wave coupling codes. In American Physical Society (APS) Meeting Abstracts, 50th Annual Meeting of the Division of Plasma Physics, page PP6.078, November 2008.

[10] COMSOL. COMSOL multiphysics, 1997-2010.

[11] S. Shiraiwa, O. Meneghini, R. Parker, P. Bonoli, M. Garrett, M. C. Kaufman, J. C. Wright, and S. Wukitch. Plasma wave simulation based on a versatile finite element method solver. Physics of Plasmas, 17(5):056119, 2010.

[12] Marco Brambilla. Slow-wave launching at the lower hybrid frequency using a phased waveguide array. Nuclear Fusion, 16(1):47-54, 1976.

[13] S. Bernabei, J.C. Hosea, C.C. Kung, G.D. Loesser, J. Rushinski, and J.R. et al. Wilson. Design of a compact lower hybrid coupler for alcator c-mod. Fusion Sci. Technol., 43:145, 2003.

[14] Y. S. Bae, C. H. Paek, M. J. Rhee, W. Namkung, M. H. Cho, S. Bernabei, and H. Park. Design of 5.0-ghz kstar lower-hybrid coupler. Fusion Engineering and Design, 65(4):569 - 576, 2003.

[15] S. Park, H. Do, J.H. Jeong, W. Namkung, M.H. Cho, H. Park, Y.S. Bae, H.L. Yang, R. Ellis, J.R. Wilson, and J. Hosea. Development status of KSTAR 5 GHz LHCD system. Fusion Engineering and Design, 85(2):197 $-204,2010$. 
[16] A. Ekedahl, M. Goniche, D. Guilhem, F. Kazarian, Y. Peysson, et al. Lower hybrid current drive in tore supra. Fusion Science and Technology, 56(3):1150-1172, October 2009.

[17] Bo-Jiang Ding, Jia-Fang Shan, Fu-Kun Liu, Yu-De Fang, Wei Wei, Zhen-Wei Wu, Zhong-Yong Chen, Han-Dong Xu, Mao Wang, Min Jiang, Gong-Rang Zhang, Feng Huang, Yan-Ping Zhao, Guang-Li Kuang, and HT-7 team. Experimental characteristics of a lower hybrid wave multi-junction coupler in the HT-7 tokamak. Chinese Physics, 15(12):2989, 2006.

[18] Lianmin Zhao, Jiafang Shan, Fukun Liu, Hua Jia, g Mao Wan, Liang Liu, Xiaojie Wang, and Handong Xu. A $2450 \mathrm{MHz} / 2 \mathrm{MW}$ lower hybrid current drive system for EAST. Plasma Science and Technology, 12(1):118, 2010.

[19] K. Kurokawa. Power waves and the scattering matrix. Microwave Theory and Techniques, IEEE Transactions on, 13(2):194 - 202, mar 1965.

[20] Roger F. Harrington. Time-Harmonic Electromagnetic Fields. Wiley-IEEE Press, New York, 2001 [1961].

[21] X. Litaudon, G. Berger-by, P. Bibet, J.P. Bizarro, J.J. Capitain, J. Carrasco, M. Goniche, G.T. Hoang, K. Kupfer, R. Magne, D. Moreau, Y. Peysson, J.-M. Rax, G. Rey, D. Rigaud, and G. Tonon. Lower hybrid wave coupling in tore supra through multijunction launchers. Nuclear Fusion, 32(11):1883, 1992.

[22] P. Jacquet, Y. Demers, G. A. Chaudron, V. Glaude, A. Côté, A. Dubé, R. Mireault, A. Robert, and L. Vachon. Microwave probe diagnostic for the lower hybrid multijunction antenna on TdeV. Review of Scientific Instruments, 68(2):1176-1182, 1997.

[23] O. Meneghini, I. Faust, D. Johnson, R. Parker, D. Terry, R. Vieira, G. Wallace, and S. Wukitch. Microwave probe diagnostic for the lower hybrid four-way-splitter antenna on alcator c-mod. In 37th EPS Conference on Plasma Physics, number P5.167, 2010.

[24] Alvin Wexler. Solution of waveguide discontinuities by modal analysis. IEEE Trans. Microwave Theory and Techniques, MTT-15(9):508-517, September 1967.

[25] F. Arndt, R. Beyer, J.M. Reiter, T. Sieverding, and T. Wolf. Automated design of waveguide components using hybrid mode-matching/numerical em building-blocks in optimization-oriented cad frameworks-state of the art and recent advances. Microwave Theory and Techniques, IEEE Transactions on, 45(5):747 -760, may 1997.

[26] Thomas Howard Stix. Waves in Plasmas. Springer, New-York, 1992 [1962].

[27] Donald Gary Swanson. Plasma Waves. Taylor \& Francis, 2nd edition, Bristol, March 2003.

[28] Stéphane Bério and Philippe Bibet. Numerical simulation of the coupling properties of advanced lower hybrid waves launchers. In 23rd EPS Conference on Controlled Fusion and Plasma Physics, Kiev, Ukrain, June 1996.

[29] Robert E. Collin. Field Theory of Guided Waves. IEEE Press, New York, 1990.

[30] John Wesson. Tokamaks. Oxford University Press, 2004.

[31] F Leuterer, F Soldner, M Brambilla, M Munich, F Monaco, M Zouhar, R Bartiromo, A Tuccillo, S Bernabei, and C Forest. Coupling of the 2x24 waveguide grill for lower hybrid waves in ASDEX. Plasma Physics and Controlled Fusion, 33(3):169, 1991.

[32] G. Rey et al. In Proceed. 15th Symp. On Fusion Technol., volume 1, page 514, 1988

[33] Ph. Bibet, G. Agarici, M. Chantant, J. J. Cordier, C. Deck, L. Doceul, A. Durocher, A. Ekedahl, Ph. Froissard, L. Garguiolo, L. Garampon, M. Goniche, P. Hertout, F. Kazarian, D. Lafon, C. Portafaix, G. Rey, F. Samaille, F. Surle, and G. Tonon. New advanced launcher for lower hybrid current drive on tore supra. Fusion Engineering and Design, 51-52:741 - 746, 2000.

[34] D. Guilhem et al. Passive active multi-junction $3.7 \mathrm{GHz}$ launcher for tore-supra long pulse experiments : Manufacturing process and tests. In Radio Frequency Power In Plasmas: Proceedings Of The 18th Topical Conference, Aip Conference Proceedings, volume 1187, pages 435-438, June 2009.

[35] A. Ekedahl et al. Validation of the iter-relevant passive-active-multijunction lhcd launcher on long pulses in tore supra. Nuclear Fusion, 50(11), November 2010.

[36] V. A. Petržílka, F. Leuterer, F.X. Söldner, L. Gianone, and R. Schubert. non-linear coupling of the lower hybrid grill in asdex. Nuclear Fusion, 31(9):1758-1767, 1991

[37] A. Ekedahl, B. Frincu, M. Goniche, J. Hillairet, and V. Petrzilka. Non-linear effects on the lh wave coupling in tore supra and impact on the lh current drive efficiency. In Radio Frequency Power In Plasmas: Proceedings Of The 18th Topical Conference, Aip Conference Proceedings, volume 1187, pages 407-410, 2009.

[38] M. Kocan, J.P. Gunn, M. Komm, J.-Y. Pascal, E. Gauthier, and G. Bonhomme. On the reliability of scrape-off layer ion temperature measurements by retarding field analyzers. Rev. Sci. Instrum., 79(073502), 2008.

[39] D. Moreau et al. Lower-hybrid current drive experiments in tore supra. Physics of Fluids B: Plasma Physics, $4(7): 2165-2175,1992$.

[40] Roberto Bilato and Marco Brambilla. FELHS code for lower-hybrid launcher coupling and near fields. In 35th EPS Plasma Physics Conf., page P5.094, 2008. 
[41] V. Pericoli-Ridolfini, Ph. Bibet, F. Mirizzi, M.L. Apicella, E. Barbato, P. Buratti, G. Calabro, A. Cardinali, G. Granucci, L. Panaccione, S. Podda, C. Sozzi, and A.A. Tuccillo. Lhcd and coupling experiments with an iter-like pam launcher on the ftu tokamak. Nuclear Fusion, 45(9):1085, 2005.

[42] R. Maggiora, D. Milanesio, and G. Vecchi. Analysis of LH launcher arrays (like the ITER one) using the TOPLHA code. In Volodymyr Bobkov and Jean-Marie Noterdaeme, editors, Radio Frequency Power In Plasmas: Proceedings Of The 18th Topical Conference, volume 1187, pages 371-374. AIP, 2009.

[43] D. Milanesio, J. Hillairet, et al. Benchmark of coupling codes (ALOHA, TOPLHA, GRILL3D) with lower hybrid antenna. In 26th Symposium on Fusion Technology (SOFT), Porto, Portugal, pages P1-153, 2010.

[44] Ph. Bibet, B. Beaumont, J.H. Belo, L. Delpech, A. Ekedahl, G. Granucci, F. Kazarian, X. Litaudon, J. Mailloux, F. Mirizzi, V. Pericoli, M. Prou, K. Rantamäki, and A. Tuccillo. Toward a LHCD system for iter. Fusion Engineering and Design, 74(1-4):419 - 423, 2005. Proceedings of the 23rd Symposium of Fusion Technology (SOFT).

[45] Alain Becoulet et al. The way towards thermonuclear fusion simulators. Computer Physics Communications, $177: 55-59,2007$.

[46] F. Imbeaux, J.B. Lister, G.T.A. Huysmans, W. Zwingmann, M. Airaj, L. Appel, V. Basiuk, D. Coster, L.-G. Eriksson, B. Guillerminet, D. Kalupin, C. Konz, G. Manduchi, M. Ottaviani, G. Pereverzev, Y. Peysson, O. Sauter, J. Signoret, and P. Strand. A generic data structure for integrated modelling of tokamak physics and subsystems. Computer Physics Communications, 181(6):987 - 998, 2010.

[47] M. Abramowitz and I.A. Stegun. Handbook of Mathematical Functions. Dover, New York, 9th edition, 1972.

\section{Appendix A. Surface plasma admittance derivations, one layer model}

\section{Appendix A.1. 2D description}

For a linear electron density profile, that is to say when $n_{e}(x)=n_{e 0}+\nabla n_{e 1} x$, it is possible to find an analytical expression of the surface admittance $\mathbb{Y}_{s}$. The dielectric parameters can be expressed in terms of normalized radial position $\xi=k_{0} x$ as:

$$
\begin{aligned}
& S(\xi)=S_{0}+\nabla S \xi \\
& D(\xi)=D_{0}+\nabla D \xi \\
& P(\xi)=P_{0}+\nabla P \xi
\end{aligned}
$$

where $S_{0}, D_{0}, P_{0}, \nabla S, \nabla D$ and $\nabla P$ are constant values.

The problem is solved considering that both the fast and slow waves, which are solutions of the propagation equation in the case of an homogeneous anisotropic plasma, exist simultaneously. In the lower hybrid frequency range, the term $\nabla S$ is negligible.

Following the classical treatment[4], the fast wave $\tilde{E}_{F}$ is characterized by $\tilde{E}_{y}=\tilde{E}_{F}$ and $\tilde{E}_{z}=0$. After some algebraic manipulation, the wave equation (1) leads in the spectral domain to:

$$
\frac{\partial^{2} \tilde{E}_{F}}{\partial \xi^{2}}+\left(\frac{\nabla D^{2}}{n_{z}^{2}-S_{0}} \xi^{2}+\frac{2 D_{0} \nabla D}{n_{z}^{2}-S_{0}} \xi+S_{0}-n_{y}^{2}-n_{z}^{2}+\frac{D_{0}^{2}-n_{y} \nabla D}{n_{z}^{2}-S_{0}}\right) \tilde{E}_{F}\left(n_{y}, n_{z}\right)=0(\mathrm{~A} .2)
$$

The general solutions of (A.2) are Whittaker functions which can be expressed using either $\mathrm{U}$ and $\mathrm{V}$ functions or $\mathrm{E}$ and $E^{*}$ complex functions [47]. leads to:

The slow wave $\tilde{E}_{S}$ is so that $\tilde{E}_{y}=-n_{y} n_{z} /\left(S-n_{z}^{2}\right) \tilde{E}_{S}, \tilde{E}_{z}=\tilde{E}_{S}$ and $\tilde{H}_{z}=0$. This assumption

$$
\frac{\partial^{2} \tilde{E}_{S}}{\partial \xi^{2}}+\left(\frac{\nabla P}{S_{0}}\left(S_{0}-n_{z}^{2}\right)\left(\xi+\frac{P_{0}}{\nabla P}\right)-n_{y}^{2}\right) \tilde{E}_{S}\left(n_{y}, n_{z}\right)=0
$$

The general solution of (A.3) is a linear combination of Airy functions Ai and Bi [47].

To choose the convenient special functions for (A.2) and (A.3), we assume that as $x \rightarrow+\infty$, the WKB's hypothesis holds and the solution of (A.2) and (A.3) have to reproduce the behavior 
of WKB waves. These considerations lead to the the physical solutions for the slow and fast waves and finally the surface admittance is found to be:

$$
\begin{aligned}
Y_{s, y y} & =\frac{j n_{z}}{S_{0}-n_{y}^{2}-n_{z}^{2}}\left(-n_{y} g_{F}+D_{0}\right) \\
Y_{s, y z} & =\frac{j}{S_{0}-n_{z}^{2}}\left[-S_{0} g_{S}+\frac{n_{y} n_{z}^{2}}{S_{0}-n_{y}^{2}-n_{z}^{2}}\left(-n_{y} g_{F}+D_{0}\right)\right] \\
Y_{s, z y} & =\frac{j}{S_{0}-n_{y}^{2}-n_{z}^{2}}\left[\left(S_{0}-n_{z}^{2}\right) g_{F}-n_{y} D_{0}\right] \\
Y_{s, z z} & =\frac{j n_{y} n_{z}}{S_{0}-n_{y}^{2}-n_{z}^{2}}\left[g_{F}-\frac{n_{y} D_{0}}{S_{0}-n_{z}^{2}}\right]
\end{aligned}
$$

where

$$
\begin{aligned}
g_{F}\left(n_{y}, n_{z}\right)=\left.\frac{\frac{\partial \tilde{E}_{F}}{\partial \xi}}{\tilde{E}_{F}}\right|_{x=0} & =\frac{\mathrm{U}^{\prime}\left(a_{W}, \eta_{W}\right)}{\alpha_{W} \mathrm{U}\left(a_{W}, \eta_{W}\right)} \text { if } S_{0}>n_{z}^{2} \\
& =\frac{\mathrm{E}^{* *}\left(a_{W}, \eta_{W}\right)}{\alpha_{W} \mathrm{E}^{*}\left(a_{W}, \eta_{W}\right)} \text { if } S_{0}<n_{z}^{2}
\end{aligned}
$$

with

$$
\begin{aligned}
& \alpha_{W}=\frac{\left|S_{0}-n_{z}^{2}\right|^{\frac{1}{4}}}{|2 \nabla D|^{\frac{1}{2}}} \\
& a_{W}=\alpha_{W}^{2}\left(n_{y}^{2}+n_{z}^{2}-S_{0}+\frac{n_{y} \nabla D}{S_{0}-n_{z}^{2}}\right) \\
& \eta_{W}==\frac{D_{0}}{\alpha_{W} \nabla D}
\end{aligned}
$$

and

$$
g_{S}\left(n_{y}, n_{z}\right)=\left.\frac{\frac{\partial \tilde{E}_{S}}{\partial \xi}}{\tilde{E}_{S}}\right|_{x=0}=\left(\frac{n_{z}^{2}-S_{0}}{S_{0}} \nabla P\right)^{\frac{1}{3}} \frac{\mathrm{Ai}^{\prime}\left(\eta_{A}\right)}{\mathrm{Ai}\left(\eta_{A}\right)}
$$

with

$$
\eta_{A}=\left[\frac{\nabla P}{S_{0}}\left(n_{z}^{2}-S_{0}\right)\right]^{\frac{1}{3}}\left(P_{0}+\frac{S_{0} n_{y}^{2}}{n_{z}^{2}-S_{0}}\right) \frac{1}{\nabla P}
$$

The expressions given here are an extension of those presented in [4] when considering $S_{0} \neq 0$ and $P_{0} \neq 0$.

\section{Appendix A.2. $1 D$ description}

In a $1 \mathrm{D}$ description, as it is implemented in SWAN code, the waveguides are supposed to be of infinite extend in $y$ direction. The coupling to the plasma is reduced to the interaction with the slow wave. Moreover, it is assumed that only the TEM mode, polarized in $z$ direction, is propagating inside the waveguides. Evanescent modes are then only $\mathrm{TM}_{n}$ modes $(n \geqslant 1)$. These assumptions 
implie that $n_{y}=0, \tilde{E}_{y}=0$ and the surface admittance $\mathbb{Y}_{s}$ reduces to the scalar $Y_{s, y z}$ (A.5). The slow wave differential equation (A.3) becomes:

$$
\frac{\partial^{2} \tilde{E}_{S}}{\partial \xi^{2}}-\frac{\nabla P}{S_{0}}\left(n_{z}^{2}-S_{0}\right)\left(\xi+\frac{P_{0}}{\nabla P}\right) \tilde{E}_{S}\left(n_{z}\right)=0
$$

which is solved (A.14) in terms of Airy function Ai and leads to the well-known 1D surface admittance:

$$
Y_{s, y z}\left(n_{z}\right)=\frac{j S_{0}}{\left(n_{z}^{2}-S_{0}\right)^{\frac{2}{3}}}\left(\frac{\nabla P}{S_{0}}\right)^{\frac{1}{3}} \frac{\mathrm{Ai}^{\prime}\left(\eta_{0}\right)}{\mathrm{Ai}\left(\eta_{0}\right)}
$$

where $\eta_{0}=\eta_{A}\left(n_{y}=0\right)$.

\section{Appendix B. Two layers model}

In this section, we derive the plasma surface admittance for the case of a plasma constituted by two consecutive linear density profiles. For the sake of explanation, let us consider a $1 \mathrm{D}$ case (slowwave only) with two layers; the $2 \mathrm{D}$ case is derived in the same way. The two layers are defined by two density gradients $\nabla n_{e 1}$ and $\nabla n_{e 2}$. The first one is a finite layer of width $d$ and the second one is supposed to be of infinitely extended (cf. figure 3). The dielectric tensor parameters vary continuously with $x$; in particular, we have $\nabla P_{1}$ for the first layer and $\nabla P_{2}$ for the second one. We define some particular values as $S_{0}=S(x=0), P_{0}=P(x=0)$ and $S_{1}=S(x=d), P_{1}=P(d=0)$. The plasma admittance expression inside the second layer at $x=d$ is similar to the case of one density gradient and is given according to equation.(A.15) by:

$$
Y_{s, d}\left(n_{z}\right)=\left.\frac{H_{y}}{E_{z}}\right|_{x=d}=\frac{j S_{1}}{\left(n_{z}^{2}-S_{1}\right)^{\frac{2}{3}}}\left(\frac{\nabla P_{2}}{S_{1}}\right)^{\frac{1}{3}} \frac{\mathrm{Ai}^{\prime}\left(\eta_{d}\right)}{\mathrm{Ai}\left(\eta_{d}\right)}
$$

with

$$
\eta_{d}=\left[\frac{\nabla P_{2}}{S_{1}}\left(n_{z}^{2}-S_{1}\right)\right]^{\frac{1}{3}}\left(\frac{P_{1}}{\nabla P_{2}}+k_{0} d\right)
$$

Inside the first layer, the solution for $\tilde{E}_{z}$ is a linear combination of both Airy functions:

$$
\tilde{E}_{z}(x=0)=\alpha \operatorname{Ai}\left(\eta_{10}\right)+\beta \operatorname{Bi}\left(\eta_{10}\right)
$$

where $\eta_{10}=\eta_{1}(\xi=0)$ with

$$
\eta_{1}(\xi)=\left[\frac{\nabla P_{1}}{S_{0}}\left(n_{z}^{2}-S_{0}\right)\right]^{\frac{1}{3}}\left(\frac{P_{0}}{\nabla P_{1}}+\xi\right)
$$

The relationship between the coefficients $\alpha$ and $\beta$ is found considering that the solutions for the transverse electric and magnetic fields at the interface of both layers $x=d$ have to be continuous. Finally, one finds the surface admittance corresponding to two successive layers:

$$
Y_{S}=\frac{\left(\frac{A i\left(\eta_{1 d}\right)}{A i\left(\eta_{10}\right)} Y_{B}-\frac{B i\left(\eta_{1 d}\right)}{B i\left(\eta_{10}\right)} Y_{A}\right) Y_{S d}+\left(\frac{B i^{\prime}\left(\eta_{1 d}\right)}{B i^{\prime}\left(\eta_{10}\right)}-\frac{A i^{\prime}\left(\eta_{1 d}\right)}{A i^{\prime}\left(\eta_{10}\right)}\right) Y_{A} Y_{B}}{\left(\frac{A i\left(\eta_{1 d}\right)}{A i\left(\eta_{10}\right)}-\frac{B i\left(\eta_{1 d}\right)}{B i\left(\eta_{10}\right)}\right) Y_{S d}+\frac{B i^{\prime}\left(\eta_{1 d}\right)}{B i^{\prime}\left(\eta_{10}\right)} Y_{B}-\frac{A i^{\prime}\left(\eta_{1 d}\right)}{A i^{\prime}\left(\eta_{10}\right)} Y_{A}}
$$

where $\eta_{1 d}=\eta_{1}(\xi=d)$. 


$$
\begin{aligned}
& Y_{A}=\frac{j S_{0}}{\left(n_{z}^{2}-S_{0}\right)^{\frac{2}{3}}}\left(\frac{\nabla P_{1}}{S_{0}}\right)^{\frac{1}{3}} \frac{\mathrm{Ai}^{\prime}\left(\eta_{0}\right)}{\mathrm{Ai}\left(\eta_{0}\right)} \\
& Y_{B}=\frac{j S_{0}}{\left(n_{z}^{2}-S_{0}\right)^{\frac{2}{3}}}\left(\frac{\nabla P}{S_{0}}\right)^{\frac{1}{3}} \frac{\mathrm{Bi}^{\prime}\left(\eta_{0}\right)}{\mathrm{Bi}\left(\eta_{0}\right)}
\end{aligned}
$$

One can extend this approach when more than two layers are considered. Suppose there are 3 layers separated at the abscissas $x=d_{1}$ and $x=d_{2}$. Starting from the further layer defined for $x \geq d_{2}$, the surface admittance $Y_{S d 2}$ at $x=d_{2}$ is calculated in a similar way that in the case of a single layer; then, the surface admittance $Y_{S d 1}$ at $x=d_{1}$ is obtained using (B.5) and the procedure is repeated to find the surface admittance $Y_{S}$ at $x=0$. 